\title{
Model of thermo-optic nonlinear dynamics of photonic crystal cavities
}

\author{
S. Iadanza, ${ }^{1,2}$ M. Clementi $\odot,{ }^{3}$ C. Hu, ${ }^{4}$ S. A. Schulz $\odot,{ }^{5}$ D. Gerace $\odot,{ }^{3}$ M. Galli, ${ }^{3}$ and L. O'Faolain ${ }^{1,2}$ \\ ${ }^{1}$ Centre for Advanced Photonics \& Process Analysis, Cork Institute of Technology, Rossa Avenue, Bishopstown, Cork T12P928, Ireland \\ ${ }^{2}$ Photonics, Tyndall National Institute, Lee Maltings Complex, Dyke Parade, Cork T12 R5CP, Ireland \\ ${ }^{3}$ Dipartimento di Fisica “A. Volta," Università degli Studi di Pavia, Pavia 21700, Italy \\ ${ }^{4}$ School of Chemistry, University College Cork, College Road, Cork T12 YN60, Ireland \\ ${ }^{5}$ School of Physics and Astronomy, University of St. Andrews, Fife KY16 9SS, United Kingdom
}

(Received 7 July 2020; revised 6 November 2020; accepted 6 November 2020; published 3 December 2020)

\begin{abstract}
The wavelength scale confinement of light offered by photonic crystal $(\mathrm{PhC})$ cavities is one of the fundamental features on which many important on-chip photonic components are based, opening silicon photonics to a wide range of applications from telecommunications to sensing. This trapping of light in a small space also greatly enhances optical nonlinearities and many potential applications build on these enhanced light-matter interactions. In order to use PhCs effectively for this purpose it is necessary to fully understand the nonlinear dynamics underlying $\mathrm{PhC}$ resonators. In this work, we derive a first principles thermal model outlining the nonlinear dynamics of optically pumped silicon two-dimensional (2D) $\mathrm{PhC}$ cavities by calculating the temperature distribution in the system in both time and space. We demonstrate that our model matches experimental results well and use it to describe the behavior of different types of PhC cavity designs. Thus, we demonstrate the model's capability to predict thermal nonlinearities of arbitrary $2 \mathrm{D} \mathrm{PhC} \mathrm{microcavities} \mathrm{in} \mathrm{any} \mathrm{material,} \mathrm{only} \mathrm{by} \mathrm{substituting} \mathrm{the}$ appropriate physical constants. This renders the model critical for the development of nonlinear optical devices prior to fabrication and characterization.
\end{abstract}

DOI: 10.1103/PhysRevB.102.245404

\section{INTRODUCTION}

The level of high sophistication in silicon microfabrication technology, and the possibility to merge the already existing electronics to photonics on the same high performance complementary metal-oxide semiconductor (CMOS) silicon chip [1], have generated recent considerable interest in silicon photonics. Silicon is becoming the leading platform for the creation of large-scale photonic integrated circuits for key enabling applications in various technology sectors, such as high-bandwidth optical interconnects and midinfrared optical sensing [2-5], offering low absorption, a high refractive index contrast and scalable mass manufacture. Recent advances in light confinement at the wavelength scale are leading to smaller and smaller high-performance resonators fabricated on silicon-on-insulator platforms. Specifically, spherical cavities [6], toroidal cavities [7,8], and photonic crystal $(\mathrm{PhC})$ cavities [9-14] offer very low optical losses [i.e., high quality $(Q)$ factors [15]], low coupling losses [16], and small mode volumes in the range of $\lambda^{3}$, and enable the investigation of cavity quantum electrodynamics [17], all-optical computing [18], lasing and parametric oscillations [19-21], and enhanced nonlinear processes $[22,23]$. The wavelength scale confinement of light in microcavities leads to a huge enhancement of the relatively small optical nonlinearities of silicon [24]. This is especially true for the thermo-optic nonlinearities, with significant power absorbed in very small volumes.

At telecom wavelengths, the dynamic behavior of an optically pumped silicon microcavity is fundamentally dependent on the thermal response of the system, governed by the heat generation associated to optical absorption-either through defect states, or via absorptive nonlinear processes such as two photon absorption (TPA) or free carrier absorption (FCA) and heat dissipation through the material. These thermal effects are much more prominent in microcavity dynamics, compared to macroscopic systems, for two main reasons: (i) heat dissipation is proportional to the surface area of the optical mode, such that the changes in temperature scale inversely with the squared size of the cavity $\left(\Delta T \propto 1 / l^{2}\right)$; (ii) the thermal time constant, which is proportional to the ratio between heat capacity and heat conductivity, scales down with the size of the cavity $\left(\tau_{t h} \propto l\right)$, making thermal processes faster. Hence, a full understanding of the thermo-optic nonlinear response of microcavities allows the most effective use of the $\mathrm{PhC}$ for the exploitation of on-chip optical bistability for a range of energy-efficient applications, as demonstrated in fast all-optical switches and memories [25,26]. To date, Carmon et al. [27] discussed the thermal response of toroidal microcavities, using two characteristic thermal time constants to fit the experimental data. These time constants correspond to a fast and a slow response, associated respectively to heat dissipation from the mode volume to the rest of the cavity and from the cavity to the surroundings, in a two thermal baths approach proposed by Ilchenko et al. [28]. The main issue with this approach is that the double exponential decay gives only an approximate description of the cavity's thermal response, while it fails to match the response accurately over all time scales, as the thermal volume of the system is dynamic. 

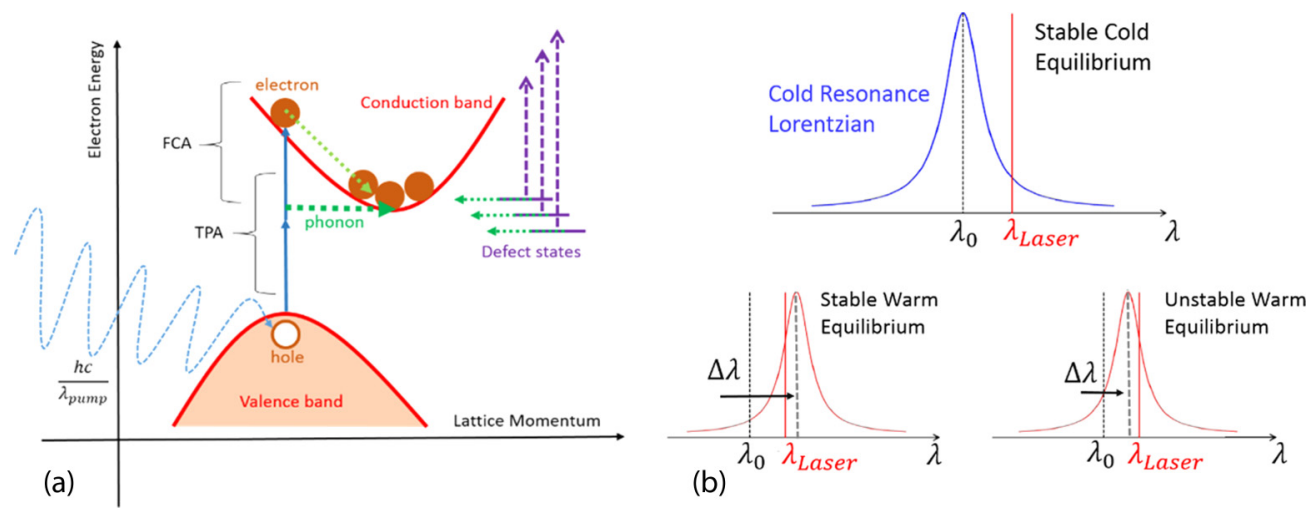

FIG. 1. (a) Schematics of the electronic energy bands in an indirect band-gap semiconductor such as silicon, depicting electron transitions related to linear and nonlinear mechanisms. (b) Schematics of all the possible equilibrium states achievable for a microcavity resonant at $\lambda_{0}$ excited by a laser at $\lambda_{\text {Laser }}$.

Furthermore, these time constants are obtained empirically by fitting the experimental data. Thus, the model is inherently only able to explain an observed response and it lacks the predictive power needed to optimize the cavity design for nonlinear optical applications.

Here, we derive a first principles thermal model describing the thermo-optic nonlinear response of optically pumped silicon $\mathrm{PhC}$ cavities. We remove the two thermal time constants approximation and eliminate all free fitting parameters but one. We calculate the temperature distribution in the surroundings of the cavity system in both space and time to correctly represent the volume of material that heats up, which increases with time as thermal energy flows outwards. This approach presents a realistic representation of the system's response and matches the experimentally observed dynamics on all relevant time scales (switch on, short and long times, including the steady state behavior). Our model eliminates the need to observe experimentally the steady state solutions prior to modeling, and it is easily applicable to evaluate the thermal nonlinearities of two-dimensional (2D) PhC microcavities in any material by switching out their respective physical constants. This enables the accurate prediction of the time-varying dynamics of optical microcavity-based devices. We demonstrate this predictive power by first modeling the behavior of different $\mathrm{PhC}$ cavity designs, and then comparing our estimates with experimental results. The ability to model nonlinear behavior prior to device fabrication and characterization is a crucial new tool for the development of nonlinear optical devices.

The paper is organized as follows: We will first describe the physical mechanisms underlying the thermo-optic dynamics of a microcavity, before introducing thermal dynamics of the system. We will then describe the experimental setup and compare the a priori model with the experimental results.

\section{FIRST-PRINCIPLES THERMO-OPTIC MODEL OF A SINGLE-MODE CAVITY}

Here we introduce a model for the nonlinear dynamics of a single-mode resonator, specifically accounting for photonelectron, photon-phonon, and electron-phonon interactions. The goal is to analyze an optically pumped silicon 2D PhC microcavity by using time-dependent classical rate equations, calculating its linear and nonlinear response. While the model is general, we initially focus on a dispersion adapted (DA) cavity [12] with far-field optimization [29,30], i.e., a distribution of $k$ vectors that allows for efficient coupling of light incident normal to the 2D cavity plane, crucial for efficient optical pumping. The detuning between the incident wavelength $\lambda_{\text {laser }}$ and the resonance wavelengths $\lambda_{0}$ affects the number of photons coupled into the cavity; the maximum coupled optical energy is achieved at the resonance condition, $\lambda_{0}=\lambda_{\text {laser }}$. When optically pumping the cavity, the following processes occur: optical energy couples into the single confined mode, and some of this energy is absorbed, e.g., through TPA, FCA, as well as defect state (linear) absorption. TPA and defect state absorption, in turn, lead to an increase in the free-carrier density and hence increased FCA. Finally, the energy absorbed through FCA or released through carrier recombination is transferred to the silicon lattice as heat. Figure 1(a) shows a schematic of the possible absorption mechanisms in the material. This heating has a double effect on the $\mathrm{PhC}$ : it changes the effective optical length of the cavity due to the thermal expansion of the lattice, and it determines an increase of the material refractive index through the thermo-optic effect. For simplicity, the thermal expansion of the material is not considered in this discussion, since the volumetric expansion coefficient of silicon is almost two orders of magnitude lower than its thermo-optic coefficient $\left(9.0 \times 10^{-6} \mathrm{~K}^{-1}\right.$ [31] compared to the $1.8 \times 10^{-4} \mathrm{~K}^{-1}$ respectively [32]) and the small changes in microcavity temperature, on the order of a few $\mathrm{K}$ as generated by the optical pumping, certainly make such an effect negligible for all practical purposes.

A change in refractive index of the material related to the carrier density and thermal processes in the $\mathrm{PhC}$ cavity leads to a shift of the resonant wavelength $\lambda_{r}$, according to the firstorder approximation:

$$
\lambda_{r}=\lambda_{0}\left[1+\left(\frac{\Delta n}{n}\right)\right]
$$

where $n$ is the refractive index of silicon and $\Delta n$ is the total refractive index change generated by the optical pumping, 
defined as

$$
\Delta n=-\left[8.8 \times 10^{-22} \Delta N_{e}+8.5 \times 10^{-18} \Delta N_{h}^{0.8}\right]+\text { TOC } \Delta T .
$$

In Eq. (2), the term in the square brackets represents the refractive index change related to the free-carrier plasma dispersion effect, expressed with Soref-Bennett constants at telecommunication wavelengths [33], and the last term represents the thermo-optic effect, in which TOC $=\frac{d n}{d T}$ is the thermo-optic coefficient of silicon and $\Delta T$ is the temperature change induced in the cavity, through both linear and nonlinear absorption. This temperature increase leads to a redshift of the cavity wavelength, which is balanced by the fact that the increased free-carrier generation also affects the refractive index of the cavity through the plasma dispersion effect, translating in a blueshifting of the cavity resonance [both accounted for in Eq. (2)].

The combination of these effects results in three possible equilibrium states for the pump-microcavity system [25], as sketched in Fig. 1(b): (i) the stable cold equilibrium, in which the cavity Lorentzian has a weak overlap with the incident laser wavelengths; therefore, insufficient optical energy is coupled into the microcavity to shift the resonant wavelength significantly [by an amount much less than the full width at half maximum (FWHM)]; (ii) the stable warm equilibrium, in which the hot resonance Lorentzian is red-detuned to the pump wavelength - a small decrease in the optical power coupled into the cavity reduces its temperature, blueshifting the resonance, which in turn increases the optical power absorbed by the cavity, redshifting it again (similarly an increase in pump power causes a small compensation to the other direction); (iii) the unstable warm equilibrium, in which the cavity Lorentzian is blue-detuned to the pump-in this configuration, a decrease in pump power leads to a decrease in the cavity temperature, resulting in the resonance drifting towards shorter wavelengths, which in turn causes a faster blueshift due to the decreasing pump power coupled into the cavity while drifting, until the system reaches the cold equilibrium state.

The main physical quantities describing the evolution dynamics of the PhC cavity are the optical energy, $u(t)$ the free-carrier number density $N(t)$, the thermal energy density $U(t)$, the heat loss rate $Q(t)$, and the temperature change $\Delta T(t)$, which can be determined by solving the following minimal set of coupled rate equations:

$$
\begin{aligned}
\frac{d u(t)}{d t}=P_{0} L-\frac{u(t)}{\tau_{q}}-\left(\frac{c}{n}\right) \sigma_{\mathrm{FCA}} u(t) N(t)-\frac{\beta}{V_{\text {mode }}}\left(\frac{c}{n}\right)^{2} u^{2}(t), \\
\frac{d N(t)}{d t}=\frac{2 \lambda_{0} c \beta n^{2}}{h V_{\text {mode }}} u^{2}(t)-\frac{N(t)}{\tau_{c}}, \\
\frac{d U(t)}{d t}=\frac{1}{V_{\text {mode }}} * \frac{u(t)}{\tau_{\text {lin }}}+\frac{2 h c}{\lambda_{\text {laser }}} * \frac{N(t)}{\tau_{c}}+\frac{\left(\frac{c}{n}\right) \sigma_{\mathrm{FCA}}}{V_{\text {mode }}} u(t) N(t) \\
+\frac{1}{V_{\text {mode }}} H(t) .
\end{aligned}
$$

Here, $d u(t) / d t, d N(t) / d t$, and $d U(t) / d t$ represent the rate of change in the optical energy, free-carrier number density, and thermal energy density within the cavity, respectively. $P_{0}$ is the optical power incident on the cavity, which in the experiment corresponds to the power of the pump laser multiplied by the coupling efficiency of the experimental setup and $L$ describes the Lorentzian line shape of the cavity, which controls the fraction of incident power coupled into the cavity (see Sec. 1 of the Appendix). The rate of change of the optical energy in the cavity [Eq. (3)] is the coupled optical power, $P_{0} L$, subtracting all the optical losses. The net loss due to linear processes (scattering and absorption) is manifested by the characteristic time constant, $\tau_{q}$, directly related to the experimental $Q$ factor of the cavity, and the nonlinear absorption represented by TPA and FCA, with $\sigma_{\mathrm{FCA}}$ being the FCA cross section, $V_{\text {mode }}=3 \times 10^{-18} \mathrm{~m}^{3}$ the optical mode volume in the cavity, $\beta$ the TPA coefficient [34], and $c$ and $n$ the speed of light in vacuum and the refractive index of the material, respectively. The dependence of the resonance linewidth on FCA and TPA is neglected in Eq. (3) as these effects are very weak compared to the pump-cavity coupling and linear absorption in the power regime considered in this work (as shown in Sec. 4 of the Appendix).

The free-carrier density at each instant [Eq. (4)] is determined by the difference between carrier generation due to TPA, related to the square of the optical energy in the cavity, and recombination characterized by the carrier lifetime $\tau_{c}$ [26]. $\lambda_{0}$ is the cavity cold resonance wavelength and $h$ is Planck's constant.

Equation (5) describes the rate of change of the thermal energy density in the cavity, determined by the balance between heat generation associated to linear and nonlinear processes and heat leaked out of the cavity by thermal conduction, which is related to the material conductivity and geometry. The heat generation is divided into three main contributions represented by the positive terms: the first term, $\frac{1}{V_{\operatorname{mode}}} \frac{u(t)}{\tau_{\text {lin }}}$, represents the thermal power density due to linear absorption, described by the characteristic time $\tau_{\text {lin }}$ discussed later. The second term, $\frac{2 h c}{\lambda_{\text {pump }}} * \frac{N(t)}{\tau_{c}}$, represents the TPA contribution to the thermal power density, in which $2 h c / \lambda_{\text {pump }}$ is the energy of the two photons, released when the carriers recombine. The third term, $\frac{\left(\frac{c}{n}\right) \sigma_{\mathrm{FCA}}}{V_{\text {mode }}} u(t) N(t)$, describes the FCA nonradiative relaxation. Finally, the last term, $\frac{1}{V_{\text {mode }}} H(t)$, describes the thermal power density flowing out of the cavity, with $H(t)$ being the heat lost from the cavity region and dissipated through the material. The calculation of $H(t)$ is performed by modeling a space- and time-dependent conductivity of the nanostructured material (cavity-PhC-bulk silicon system) which is discussed in detail in the next section.

\section{THERMAL DYNAMICS OF THE SINGLE MODE CAVITY}

Equation (5) shows that the heat generation at each instant is counteracted by the heat flowing out of the warming cavity, represented by the term $H(t)$. In this section, the heat dissipation dynamics, governed by $H(t)$, are discussed in detail.

Heat is generated within a small region of the system, specifically the volume of the mode confined in the cavity $\left(V_{\text {mode }}\right)$, which we will from now on refer to as the "warming zone." To describe the heat leaked from this warming zone 


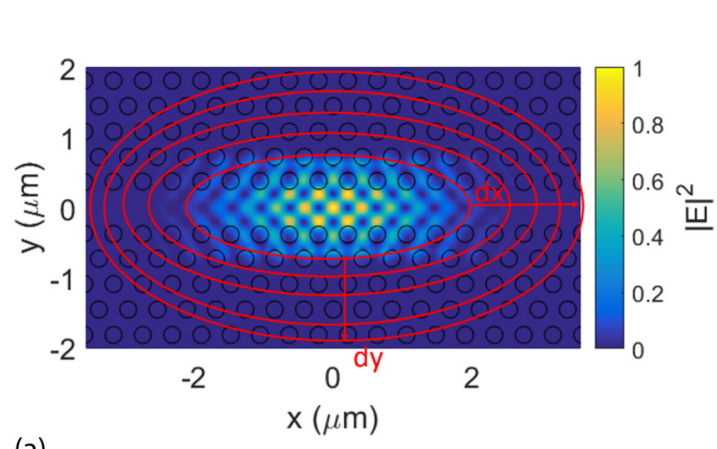

(a)

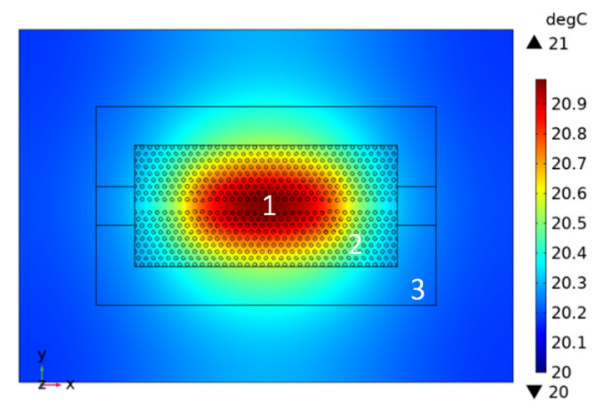

(b)

FIG. 2. (a) Optical mode confinement of a silicon DA PhC cavity calculated with the finite difference time domain (FDTD) method. Red concentric ellipses represent a scheme showing the spatial subdivision of the PhC used for the thermal calculation. (b) Heat generated by the optical mode confined in the DA PhC cavity calculated with the finite element method (FEM). Three different regions characterized by a different thermal conductivity are highlighted: (1) confinement of the mode in the cavity, (2) rest of the photonic crystal, (3) slab of unpatterned silicon.

we divide the system into many concentric elliptical portions of space centered on the PhC cavity and enclosing progressively bigger portions of material, as shown in Fig. 2. Each consecutive ellipse has an $x_{i}$ and $y_{i}$ axis wider by an increment $(d x, d y)$ compared to the previous ellipse:

$$
\begin{aligned}
& x_{i+1}=x_{i}+d x \\
& y_{i+1}=y_{i}+d y
\end{aligned} .
$$

A convergence test was performed using different increment values in order to verify the correct resolution of the calculations while optimizing their speed, with a uniform increment $d x=d y=150 \mathrm{~nm}$ and a time step $d t=50 \mathrm{ps}$ providing the perfect balance (Sec. 2 of the Appendix). This increment is used for the remainder of this work. Therefore, each concentric ellipse delimits an expanding donut-shaped surface area, $d x$ wide and $h_{S i}$ thick, with $h_{S i}$ being the thickness of the silicon layer on which the cavity is fabricated. The optical mode confined in the cavity, of approximated elliptical shape, heats up an expanding elliptical partition of the material with lower and lower eccentricity until the shape converges into a circle. In order to calculate the heat leaked of the cavity $H(t)$ at each time step, the model evaluates the temperature distribution of the material at each instant in time, starting from a uniform temperature distribution, with $T(t=0)=20^{\circ} \mathrm{C}$ in each donut (room temperature).

The heat generated by the optical absorption of the pump in $V_{\text {mode }}$ at the center of the cavity flows out through the corresponding surface and into the next donut-shaped portion of material with a rate dependent on the local thermal conductivity and temperature gradient, following Fourier's law of heat conduction, rearranged in the following equations:

$$
\begin{gathered}
U_{\operatorname{Total}_{i}}(t)=U_{i n_{i}}(t)+H_{i}(t), \\
H_{i}(t)=-K_{i} A_{i}\left(\frac{T_{i}-T_{i+1}}{d x}\right) d t .
\end{gathered}
$$

The subscript $i$ refers to the $i$ th donut-shaped portion of material, with the $V_{\text {mode }}$ at the center of the PhC cavity being the first one, i.e., $i=1 . K_{i}$ and $A_{i}$ are the thermal conductivity and surface area of the corresponding $i$ th donut, respectively. The temperature gradient is approximated in each donut by the difference between the local temperature $T_{i}$ and the temperature in the colder adjacent donut, $T_{i+1}$, over the incremental thickness of donut, $d x$ :

$$
\frac{d T}{d x} \approx \frac{\left(T_{i}-T_{i+1}\right)}{d x}
$$

$U_{\text {Total }_{i}}(t)$ and $U_{i n_{i}}(t)$ represent respectively the total heat and the heat flowing into the corresponding donut-shaped portion of material. All these terms are dependent on the geometry and the material properties of the system, and are modeled by dividing the system in three regions, as follows:

The first region is the $\mathrm{PhC}$ cavity in which the optical mode is confined, represented by $V_{\text {mode }} \sim A_{\text {cavity }} * h_{\mathrm{Si}}$. Here $A_{\text {cavity }}$ is the area of the cavity, approximated to the area of an ellipse whose $x$ and $y$ dimensions extend to the points at which the electric field of the confined mode, $E(x)$ and $E(y)$, decays to $1 / e$. This translates into an ellipse with sizes $x=5.0 \mu \mathrm{m}$ and $y=2.5 \mu \mathrm{m}$; see Fig. 2(b). The height is fixed as $h_{\mathrm{Si}}=$ $220 \mathrm{~nm}$, which corresponds to the thickness of the silicon layer of the silicon-on-insulator (SOI) chip, on which the $\mathrm{PhC}$ cavity is fabricated. In this region, the absorbed optical energy acts as the increasing thermal energy at each instant, $U_{i n_{1}}(t)$, that is the heat flowing in the first zone. This zone is characterized by the thermal conductivity of bulk silicon $(K=130 \mathrm{~W} / \mathrm{mK})$ and the surface area for the heat transfer is $A_{1}=2 P_{1} h_{S i}$, corresponding to the $2 \mathrm{D}$ elliptical donut with perimeter $2 P_{1}$, calculated with the Ramanujan approximation [35], and $h_{\mathrm{Si}}=220 \mathrm{~nm}$. The cavity and surrounding $\mathrm{PhC}$ structure are totally encapsulated in air due to the removal of the $\mathrm{SiO}_{2}$ sacrificial layer underneath, through a hydrofluoric acid (HF) isotropic etching process. Due to the very low thermal conductivity of air as compared to silicon and the negligible contribution from convection, we neglect heat lost through top and bottom surfaces of this zone. Hence, the total heat present at each instant in this zone, is evaluated as follows:

$$
\begin{aligned}
U_{i n_{1}}(t)= & \left\{\left(\frac{u(t)}{\tau_{\text {lin }}}\right)+\left(\frac{2 h c}{\lambda_{\text {laser }}} \frac{N(t)}{\tau_{c}}\right)+\left[\left(\frac{c}{n}\right) \sigma_{\mathrm{FCA}} u(t) N(t)\right]\right\} \\
& \times d t
\end{aligned}
$$




$$
\begin{gathered}
H_{1}(t)=-K_{\text {bulk }} A_{1}\left(\frac{T_{1}-T_{2}}{d x}\right) d t, \\
U_{\operatorname{Total}_{1}}(t)=U_{i n_{1}}(t)+H_{1}(t) .
\end{gathered}
$$

The surface area $A_{1}$ and heat capacity $C_{p_{1}}$ of this portion of material are

$$
\begin{gathered}
A_{1}=\pi\left[3\left(x_{1}+y_{1}\right)-\sqrt{\left(3 x_{1}+y_{1}\right)\left(x_{1}+3 y_{1}\right)}\right] h_{\mathrm{Si}}, \\
C_{p_{1}}=\rho c_{p} V_{1}=\rho c_{p} \pi\left(x_{1} y_{1}\right) h_{\mathrm{Si}} .
\end{gathered}
$$

Literature values have been used for the density and specific heat at constant pressure of silicon, $\rho=2.33 \mathrm{~g} / \mathrm{cm}^{3}$ and $c_{p}=0.7 \mathrm{~J} / \mathrm{gK}$ [36-38]. From the heat balance, the local time-dependent temperature increase due to positive thermal balance is calculated in this first zone by using the definition of heat for a solid:

$$
\Delta T_{1}(t)=\frac{U_{\mathrm{Total}_{1}}(t)}{C_{p_{1}}} .
$$

The increased temperature at each time step of the calculation, $T_{1 \text { new }}(t)$, is the temperature of the material in this zone calculated in the previous time step plus $\Delta T_{1}(t)$ :

$$
T_{1_{\text {new }}}(t)=T_{1}(t)+\Delta T_{1}(t) .
$$

The second zone corresponds to the first $d x$-wide donutshaped region surrounding the $\mathrm{PhC}$ cavity, which is part of the $\mathrm{PhC}$ cladding and therefore characterized by the physical properties of the membraned and patterned $\mathrm{PhC}$ region. Hence, a transition of the conductivity of the material occurs between zone 1 (bulk silicon) and zone 2 (patterned silicon). To map the temperature and evaluate the heat balance in this zone, the following equations are used:

$$
\begin{gathered}
U_{\mathrm{Total}_{2}}(t)=K_{\mathrm{bulk}} A_{1}\left(\frac{T_{1}-T_{2}}{d x}\right) d t-K_{\mathrm{PhC}} A_{2}\left(\frac{T_{2}-T_{3}}{d x}\right) d t \\
A_{2}=\pi\left[3\left(x_{2}+y_{2}\right)-\sqrt{\left(3 x_{2}+y_{2}\right)\left(x_{2}+3 y_{2}\right)}\right] h_{\mathrm{Si}} \\
C_{p_{2}}=\rho c_{p} V_{2}=\rho c_{p} \pi\left[\left(x_{2}+y_{1}\right) d x\right] h_{\mathrm{Si}} .
\end{gathered}
$$

At each time step, the heat flow out of the previous zone (zone 1) enters the current zone (zone 2), effectively increasing the temperature in the region, counteracted by heat conduction out of this zone (to zone 3 ), characterized by the conductivity of the membraned $\mathrm{PhC}, K_{\mathrm{PhC}}=90 \mathrm{~W} / \mathrm{mK}$ [39-41]. We note that due to the nanostructured nature of this region both the heat capacity and conductivity differs from

\begin{tabular}{|c|c|}
\hline Parameter of model & Value used in the calculations \\
\hline$V_{\text {mode }}(\mathrm{DA})$ & $3 \times 10^{-18} \mathrm{~m}^{3}$ \\
\hline$Q$ factor (DA) & 35000 \\
\hline$\lambda_{0}(\mathrm{DA})$ & $1548.447 \mathrm{~nm}$ \\
\hline$V_{\text {mode }}(L 3)$ & $2 \times 10^{-18} \mathrm{~m}^{3}$ \\
\hline$Q$ factor $(L 3)$ & 16000 \\
\hline$\lambda_{0}(L 3)$ & $1525.964 \mathrm{~nm}$ \\
\hline$\tau_{\operatorname{lin}}$ & $200 \mathrm{ps}$ \\
\hline$\tau_{c}$ & $150 \mathrm{ps}$ \\
\hline $\mathrm{TOC}_{\mathrm{Si}}$ & $1.8 \times 10^{-4} \mathrm{~K}^{-1}$ \\
\hline$n_{\mathrm{Si}}$ & 3.48 \\
\hline$\beta$ & $8 \times 10^{-12} \mathrm{~m} / \mathrm{W}$ \\
\hline$\sigma_{\mathrm{FCA}}^{\mathrm{Si}}$ & $2 \times 10^{-21} \mathrm{~m}^{2}$ \\
\hline$d x$ & $150 \mathrm{~nm}$ \\
\hline$d t$ & $50 \mathrm{ps}$ \\
\hline$\rho_{\mathrm{Si}}$ & $2.33 \mathrm{~g} / \mathrm{cm}^{3}$ \\
\hline$\rho_{\mathrm{SiO} 2}$ & $2.17 \mathrm{~g} / \mathrm{cm}^{3}$ \\
\hline$L_{\mathrm{PhC}}$ & $40 \mu \mathrm{m}$ \\
\hline$h_{\mathrm{Si}}$ & $0.22 \mu \mathrm{m}$ \\
\hline$h_{\mathrm{SiO}_{2}}$ & $2.0 \mu \mathrm{m}$ \\
\hline$K_{\mathrm{si}}$ & $130 \mathrm{~W} / \mathrm{cmK}$ \\
\hline$K_{\mathrm{PhC}}$ & $90 \mathrm{~W} / \mathrm{cmK}$ \\
\hline$c_{p}^{\mathrm{Si}}$ & $0.70 \mathrm{~J} / \mathrm{gK}$ \\
\hline$c_{p}^{p} \mathrm{SiO}_{2}$ & $0.68 \mathrm{~J} / \mathrm{gK}$ \\
\hline$x, y(\mathrm{DA})$ & $5.0 \mu \mathrm{m}, 2.5 \mu \mathrm{m}$ \\
\hline$x, y(\mathrm{~L} 3)$ & $3.0 \mu \mathrm{m}, 2.0 \mu \mathrm{m}$ \\
\hline$T_{\text {room }}$ & $20^{\circ} \mathrm{C}$ \\
\hline
\end{tabular}
that of bulk silicon (see Table I). Once again,

$$
\begin{gathered}
\Delta T_{2}(t)=\frac{U_{\text {Total } 2}(t)}{C_{p_{2}}}, \\
T_{2_{\text {new }}}(t)=T_{2}(t)+\Delta T_{2}(t) .
\end{gathered}
$$

The third region corresponds to the rest of the material: the ensemble of all the adjacent donuts after zone 2, including the rest of the $\mathrm{PhC}\left(\mathrm{a} 20 \times 20 \mu \mathrm{m}^{2}\right.$ square, $220 \mathrm{~nm}$ thick) and
TABLE I. Parameters of the model used for the calculations.

a $500 \times 500 \mu \mathrm{m}^{2}$ of bulk, i.e., unpatterned, silicon, $220 \mathrm{~nm}$ thick. The $h_{\mathrm{SiO} 2}=2-\mu \mathrm{m}$-thick layer of silica is still present underneath the unpatterned silicon after the $\mathrm{PhC}$, and is integrated into the heat transfer discussion. The heat balance of all of these donuts is described by the following equations:

$$
\begin{aligned}
U_{\mathrm{Total}_{i}}(t)= & K_{i-1} A_{i-1}\left(\frac{T_{i-1}-T_{i}}{d x}\right) d t-K_{i} A_{i}\left(\frac{T_{i}-T_{i+1}}{d x}\right) d t \\
& -K_{\mathrm{SiO}_{2}} A_{\mathrm{SiO}_{2} i}\left(\frac{T_{i}-T_{i+1}}{h_{\mathrm{SiO}_{2}}}\right) d t \\
A_{i}= & A_{\mathrm{Si}_{i}}+A_{\mathrm{SiO}_{2} i} \\
= & \pi\left[3\left(x_{i}+y_{i}\right)-\sqrt{\left(3 x_{i}+y_{i}\right)\left(x_{i}+3 y_{i}\right)} h_{\mathrm{Si}}\right. \\
& +\pi\left[\left(x_{i}+y_{i-1}\right) d x\right] \\
C_{p_{i}}= & \rho c_{p} V_{i}+\rho_{\mathrm{SiO}_{2}} c_{p \mathrm{SiO}_{2}} V_{\mathrm{SiO}_{2} i} \\
= & \pi\left(\rho c_{p} h_{\mathrm{Si}_{1}}+\rho_{\mathrm{SiO}_{2}} c_{p \mathrm{SiO}_{2}} h_{\mathrm{SiO}_{2}}\right)\left[\left(x_{i}+y_{i-1}\right) d x\right]
\end{aligned}
$$

where the density and the specific heat of silica assume the literature values of $\rho_{\mathrm{SiO}_{2}}=2.17 \mathrm{~g} / \mathrm{cm}^{3}$ and $c_{\mathrm{SiO} 2}=0.7 \mathrm{~J} / \mathrm{gK}$ respectively $[42,43]$. In these equations, the thermal conductivity $K_{i}$ takes the value for membraned silicon in the $\mathrm{PhC}$ and the value for bulk silicon in the rest of the space, according to the conditions

$$
K_{i}\left(x_{i}\right)=\left\{\begin{array}{l}
90 \mathrm{~W} / \mathrm{mK}, x_{i}<20 \mu \mathrm{m} \\
130 \mathrm{~W} / \mathrm{mK}, x_{i} \geqslant 20 \mu \mathrm{m}
\end{array}\right.
$$




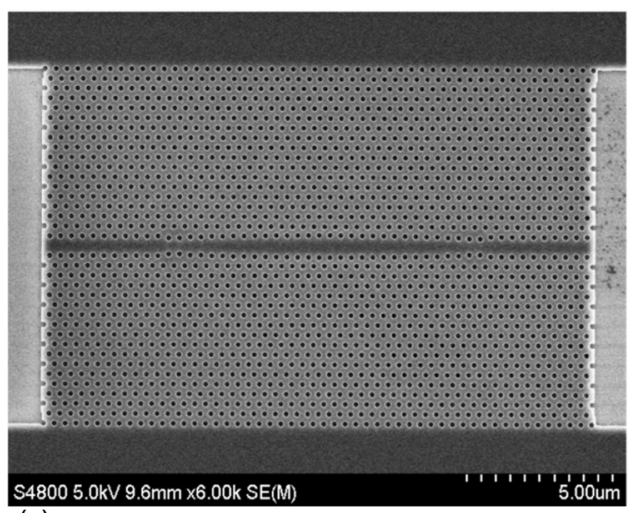

(a)

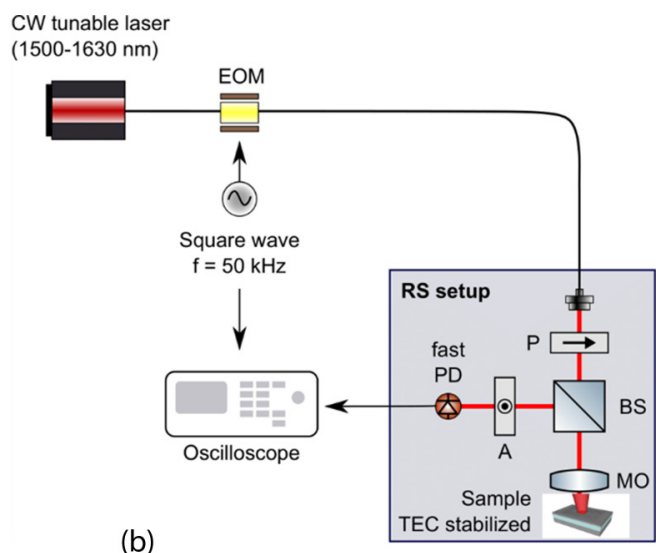

(b)

FIG. 3. (a) SEM image of a fabricated silicon DA PhC cavity and (b) schematics of the resonant scattering setup used for the measurement of the DA cavity thermo-optic dynamics. $A$ and $P$ represent two orthogonal polarizers, BS is a nonpolarizing beam-splitter, EOM is an electro-optic modulator, $\mathrm{PD}$ is a photodetector, and MO is the microscope objective used to focus the laser onto the cavity.

and $K_{\mathrm{SiO}_{2}}=1.5 \mathrm{~W} / \mathrm{mK}$, taken from the literature [40]. Furthermore, the surface area and thickness of silica have null values underneath the $\mathrm{PhC}$, as defined below:

$$
\begin{aligned}
& A_{\mathrm{SiO}_{2}}\left(x_{i}\right)=\left\{\begin{array}{c}
0, x_{i}<20 \mu \mathrm{m} \\
A_{\mathrm{SiO}_{2 i},}, x \geqslant 20 \mu \mathrm{m}
\end{array},\right. \\
& h_{\mathrm{SiO} 2}\left(x_{i}\right)=\left\{\begin{array}{c}
0, x<0 \\
h_{\mathrm{SiO} 2}, x \geqslant 0
\end{array}\right.
\end{aligned}
$$

The temperature distribution in this in this portion of material is then evaluated with

$$
\begin{gathered}
T_{i_{\text {new }}}(t)=T_{i}(t)+\Delta T_{i}(t), \\
\Delta T_{i}(t)=\frac{U_{\text {Total } i_{i}}(t)}{C_{p_{i}}} .
\end{gathered}
$$

From the time and space dependent temperature mapping of the system just discussed, we now extract, at each time step, the total temperature increase of the cavity from its room temperature initial condition, $\Delta T(t)=T_{1_{\text {new }}}-T_{\text {room }}$, and use it in the evaluation of Eq. (2). The total heat lost from the cavity at each time step, $H_{1}(t)$, is instead for the calculation of Eq. (5).

\section{EXPERIMENTAL SETUP}

The DA photonic crystal cavity was exposed on ZEP 520 A resist by means of electron-beam lithography and the pattern transferred into the 220-nm-thick silicon layer of an SOI wafer with a $2-\mu$ m-thick buried oxide layer though dry etching in a fluorine-based $\left(\mathrm{CHF}_{3} / \mathrm{SF}_{6}\right)$ plasma. Figure 3(a) shows the scanning electronic microscope (SEM) image of the fabricated microcavity. We pumped the $2 \mathrm{D} \mathrm{PhC}$ photonic crystal cavity using a continuous-wave laser beam, tunable over 1.5-1.6- $\mu \mathrm{m}$ spectral range, with a $\pm 2 \mathrm{pm}$ tuning accuracy, in order to precisely select the pump wavelengthcavity mode detuning, by the resonant scattering technique [29]. The pump laser is launched though a polarizer $(P)$ and then focused onto the sample through an objective lens. The orientation of $P$ is such that the incident light is polarized at $45^{\circ}$ to the $\mathrm{PhC}$ cavity. Therefore, the light reflected/scattered back from the cavity is cross polarized to the excitation polarization. The confocal geometry of the setup allows the collection of this scattered/reflected light, which is redirected towards a second polarizer ( $A$, orthogonal to $P$ ) through a nonpolarizing beam splitter (BS), and measured by the detector (PD) as shown in Fig. 3(b).

In this way, the $P$-polarized laser beam has a component that couples to the cavity mode and the other that reflects back at the air-silicon interface of the SOI chip; the latter component is stopped by the cross-polarizer while a portion of the former acquires polarization $A$, passes through the second polarizer, and is collected by the detector. The cavity mode demonstrates itself as a higher intensity peak on the small nonresonant continuum background signal, which is suppressed by the second polarizer $A$. For the measurement of the thermo-optic dynamics of the cavity response, the pump beam is modulated in amplitude between $0 \%$ and $100 \%$ level by an electro-optical modulator (EOM) driven by an arbitrary function generator (square wave modulation, $f=$ $50 \mathrm{kHz}, \mathrm{DC}=50 \%$ ) so that the cavity responds according to the ON/OFF switching of the laser.

\section{NUMERICAL AND EXPERIMENTAL RESULTS}

The PhC cavity operates as a narrow wavelength filter and has the fundamental mode resonant at wavelength $\lambda_{0}=$ $1548.447 \mathrm{~nm}$, with a measured $Q$ factor $Q_{\text {meas }}=35000$, which manifests as a Lorentzian peak with FWHM $=\frac{\lambda_{0}}{Q_{\text {meas }}}=$ $0.044 \mathrm{~nm}$. The characteristics of the silicon 2D PhC were measured experimentally by scanning the pump over a broad range of wavelengths at different laser power and collecting the resonant scattering response of the cavity, as seen from the experimental spectra in Fig. 4(a).

As the laser wavelength is swept across the resonance, linear absorption, TPA, and FCA occur (as discussed above), and the cavity resonance wavelength drifts away from the pump wavelength. At a certain value, absorption reaches its maximum and if the laser is tuned further, the resonance is lost. This self-tuning of the cavity resonance gives rise to the asymmetry observed in Fig. 4. This effect is well known in the literature [27,44] and following standard practice, we refer 

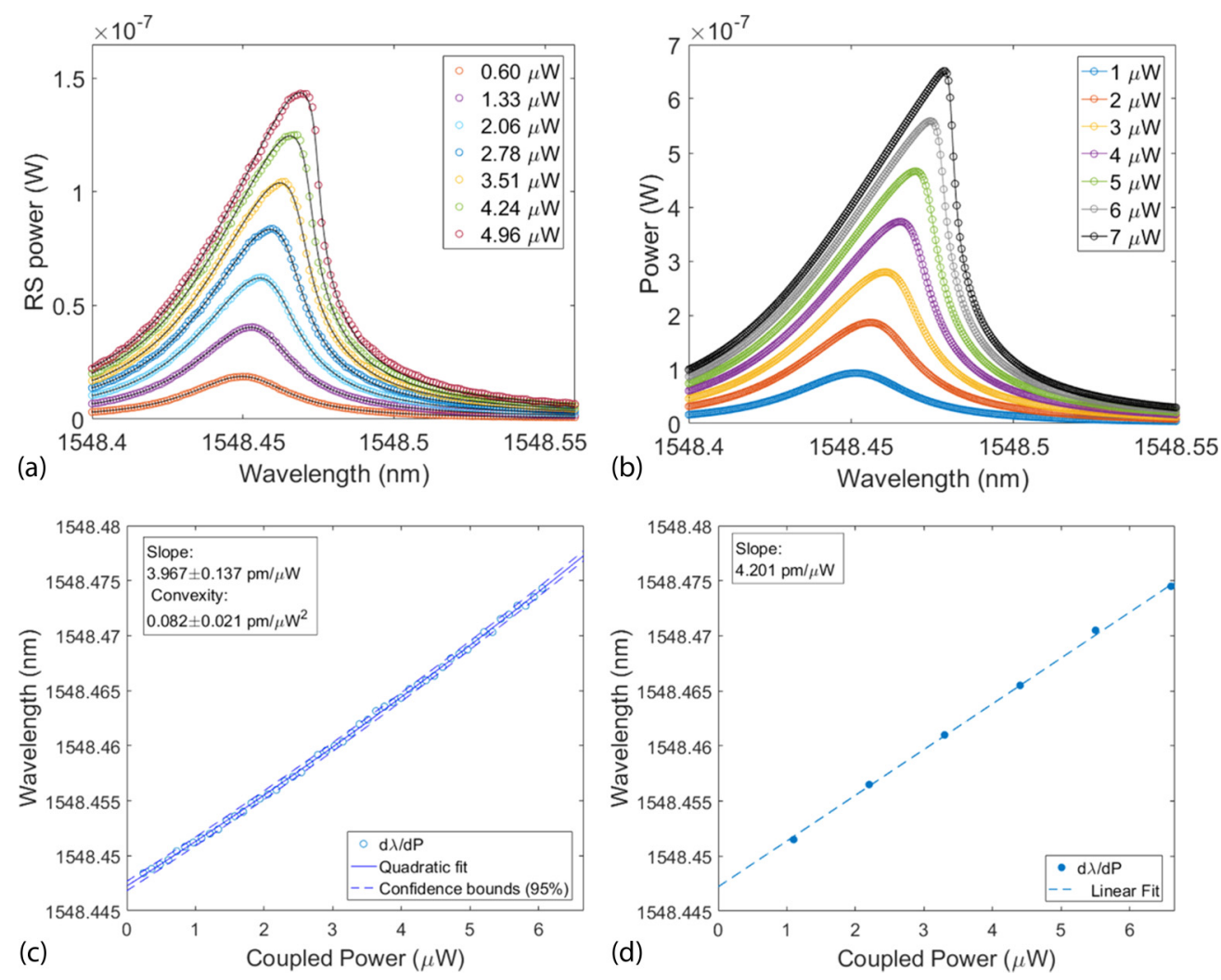

FIG. 4. (a) The experimental response of the DA cavity generated by sweeping the wavelength of the exciting laser: the optical power collected from the cavity is plotted against wavelength. (b) Plot of the calculated response of the DA cavity generated by sweeping the wavelength of the exciting laser. (c) Plot of the experimental wavelength shift of the cavity resonance with coupled power of the laser with quadratic fit and (d) the corresponding plot calculated with the model with a $\tau_{\text {lin }}=200 \mathrm{ps}$.

to the resonance wavelength of the warm cavity as the "hot cavity."

The hot cavity resonance wavelength redshifts with increasing pump power and its trend is plotted in Fig. 4(c). The almost linear trend of this experimental resonance wavelength redshift shows that the dominating contribution to the cavity response in this laser power regime is linear absorption related to surface and defects states, with TPA and FCA processes being more prominent in the curves representing the highest injected powers, which become increasingly asymmetric due to increased self-tuning of the cavity resonance at high powers (bistable regime [26,27]), speeding up the drifting of the cavity resonance. The cavity response peak is in fact sharper at higher powers as the increasingly dominant TPA and FCA processes induce a quadratic wavelength drift of the cavity cold resonance. This leads to a faster pump-cavity detuning (smaller and smaller overlap between the pump line and the cavity Lorentzian), which in turn translates into an abrupt decay of the power coupled into the cavity.

There is no literature value for the linear absorption coefficient, since this parameter is strongly dependent on the particular fabrication process used, for example, on the surface passivation. Thus, we use linear absorption as the only fitting parameter by matching the linear wavelength redshift of the cavity resonance, $d \lambda / d P$, measured to be around $4 \mathrm{pm} / \mu \mathrm{W}$ [slope of the curve in Fig. 4(c)], which corresponds to a linear absorption characterized by a time constant $\tau_{\text {lin }}=$ 200 ps. This is the value used in Eqs. (5) and (9). The model reproduces the experimental curves as shown in Fig. 4(b) and reproduces the linear dependence of the resonance wavelength shift with increasing pump power; see Fig. 4(d). Relevant nonlinear trends of the cavity dynamics become noticeable with coupled powers higher than these values, as shown by the model (Sec. 3 of the Appendix).

The thermo-optic dynamics of the pump-cavity system are then studied by red-detuning the pump laser with respect to the cavity cold resonance, such that, when the laser switches on, the cavity absorbs a portion of the incoming radiation, heats up, and redshifts towards the pump wavelength through the thermo-optic effect, while gradually increasing the portion of the light coupled into it, until it reaches the hot equilibrium state. We refer to the time the cold resonance takes to reach the pump wavelength as the switch-on time, which is strongly dependent on the power coupled into the cavity, which in turn relates to the pump-cavity detuning. Figure 5(a) shows the experimental switch-on curves at constant pump power for different pump-cavity detuning. In this plot, the switch-on time is defined as the time at which the optical power in the cavity reaches its maximum value. As expected, an increased detuning corresponds to longer switch-on times. This time-varying behavior of the cavity response, in both the rapid switch on and slow approach to the steady state, is accurately captured by the model, as shown in Fig. 5(b). The calculated curves accurately match the experimental ones at the same detuning and pump power. The detuning in Fig. 5 is expressed in pm; 

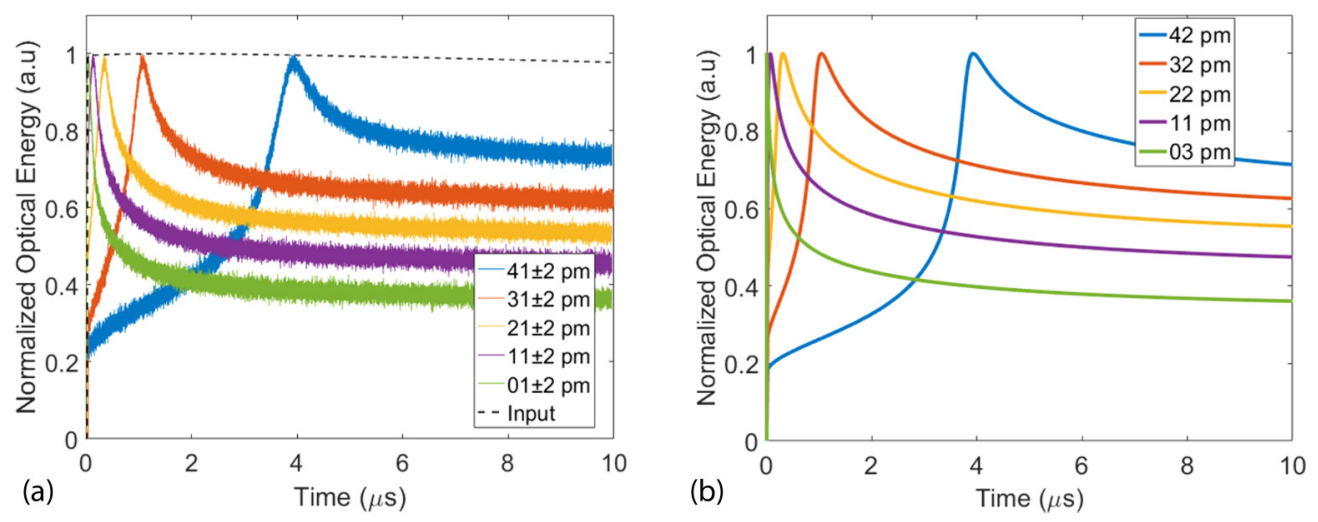

FIG. 5. (a) Experimental dynamic response of the DA cavity: the normalized optical energy collected from the DA cavity is plotted against time. The different colored curves represent the cavity response with different laser-cavity detuning $\lambda_{\text {Laser }}-\lambda_{0}$, the black dashed line represents the experimental input signal. (b) Dynamics of the DA cavity calculated with the model described in this work. The different laser-cavity detuning $\lambda_{\text {Laser }}-\lambda_{0}$ is matched to the experimental measurements for the calculations. Normalization has been carried out by dividing each raw curve by its maximum value, so that all the peaks were reaching unity.

the same values converted in fractions of the bare DA cavity linewidth $(44 \mathrm{pm})$ are $41 \mathrm{pm}=0.9318 \mathrm{FWHM}$, $31 \mathrm{pm}=0.7045$ FWHM, $\quad 21 \mathrm{pm}=0.4773$ FWHM, $11 \mathrm{pm}=0.25 \mathrm{FWHM}, \quad 1 \mathrm{pm}=0.0227 \mathrm{FWHM}$ with an error of $\pm 2 \mathrm{pm}=0.0455 \mathrm{FWHM}$ for the experiment, and $\quad 42 \mathrm{pm}=0.9545 \mathrm{FWHM}, \quad 32 \mathrm{pm}=0.7273 \mathrm{FWHM}$, $22 \mathrm{pm}=0.5 \mathrm{FWHM}, \quad 11 \mathrm{pm}=0.25 \mathrm{FWHM}, \quad 3 \mathrm{pm}=$ 0.0683 FWHM for the simulations

To show the generality of the model, we use it to predict the time-dependent response of a different optical system, consisting of an optically pumped L3 PhC cavity with different resonant modes and $Q$ factor. All parameters in the model are retained (and retain their value), with the exception of $V_{\text {mode }}$ and $A_{\text {cavity }}$, which are adapted the match the mode of the L3 PhC geometry, as reported in Fig. 6(a). The good match between the calculated results and the experimental curves, as shown in Fig. 6(b), validates the model as a means of predicting the thermo-optic dynamics of any laser-driven single-mode 2D PhC cavity system. The detuning in Fig. 6 is expressed in pm; the same values converted in fractions of the bare $L 3$ cavity linewidth $(95 \mathrm{pm})$ are $66 \mathrm{pm}=$ 0.6947 FWHM, $56 \mathrm{pm}=0.5895$ FWHM, $46 \mathrm{pm}=0.4842$ FWHM, $36 \mathrm{pm}=0.379$ FWHM, $26 \mathrm{pm}=0.2737$ FWHM, and $\quad 67 \mathrm{pm}=0.7053$ FWHM,$\quad 58 \mathrm{pm}=0.6105$ FWHM, $45 \mathrm{pm}=0.4737$ FWHM, $\quad 37 \mathrm{pm}=0.3895$ FWHM, $26 \mathrm{pm}=0.2737 \mathrm{FWHM}$ for the simulations

A related approach was used for slow light $\mathrm{PhC}$ waveguides in Refs. [45,46]. Empirical parameters describing the surface roughness were determined in the first paper [43] and used to make predictions that were validated in a separate fabrication run [46] showing that, for a given fabrication process, the fabrication quality remains broadly consistent in time. We expect $\tau_{\text {lin }}$ to be a characteristic of a given fabrication process and to therefore also stay relatively constant over time (so long as no dramatic changes are made to the fabrication recipe).

Furthermore, we also show calculations of the temperature distribution for different cavity configurations. Figure 7 depicts a schematics of the 2D PhC cavity in two configurations: (a) shows the cavity patterned on the SOI with no trenches etched around it to delimit the PhC; (b) shows the cavity pat- terned on the SOI with some trenches etched into the silicon slab to isolate the $\mathrm{PhC}$ from the rest of the silicon, apart from four small silicon bridges.

In this work, we use the model to study the evolution of heat generation in the system. We express this as a heated volume that expands over time, as the temperature spatial profile (i.e., the spatial coordinate at which the temperature of the material decays to $1 / e^{2}$, here expressed as $r_{t}$ ) of the material enlarges. In a 3D view, $r_{t}$ is the radius of the red circumference centered on the cavity that defines the points of the material where the temperature decayed to $1 / e^{2}$ (the borders of the temperature spatial profile) at each time step, shown in Fig. 7. As time passes, the cavity heats up and the temperature distribution broadens, which translates into an increase of $r_{t}$ depicted by an enlarging red circumference.

Figure 8(a) shows the calculated dynamic behavior of the thermal volume for the cavity used in the experimental work (i.e., the temperature spatial profile change with time), shown schematically in Fig. 7(a), where the cavity is surrounded directly by the unpatterned silicon region. These results are compared to simulated results corresponding to the same cavity in the case where it is insulated by air and connected to the unpatterned silicon region through smaller and smaller silicon bridges; see the schematic in Fig. 7(b). The calculations have been carried out with the silicon bridges having fixed length and thickness of 5 and $0.22 \mu \mathrm{m}$ respectively, and a varying lateral width of $2,4,6,10,12,22,32$, and $42 \mu \mathrm{m}$.

A clear trend emerges, with a slower expanding thermal volume related to the progressively lower thermal conductivity as the size of the silicon bridges decreases. These curves indicate that the temperature spatial profiles, expressed with the radius $r_{t}$, are broadening more and more slowly the smaller the silicon bridges are (that is, heat is being confined for longer time in the $\mathrm{PhC}$ ), down to the case of the $2-\mu \mathrm{m}$-wide bridge, where the temperature profile is enlarging very slowly up to $\sim 6.3 \mu \mathrm{s}$, until heat is finally able to diffuse in the silicon slab, leading to a faster increase of $r_{t}$. Figure 8(c) show the temperature space profiles for the different types of cavities at the last instant (after $10 \mu$ s of optical pumping). The more thermally insulated the cavity is (i.e., smaller silicon bridge) 


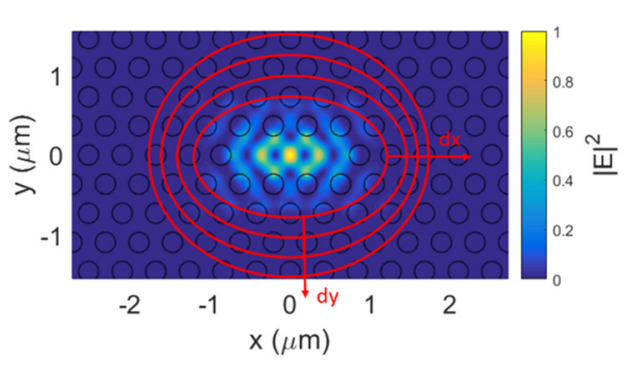

(a)

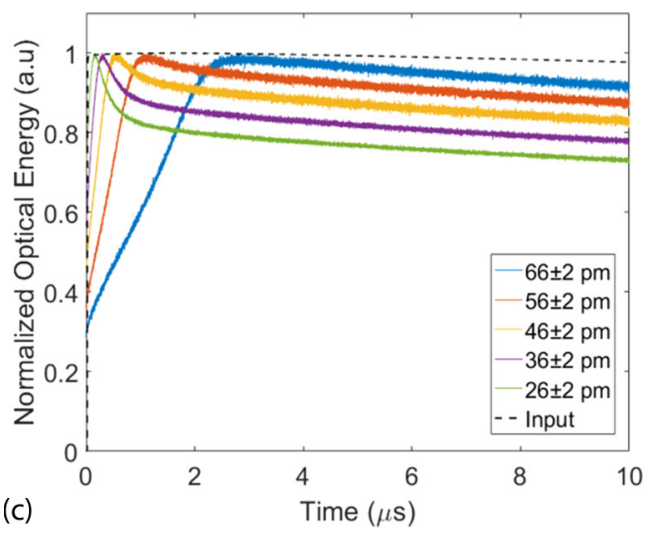

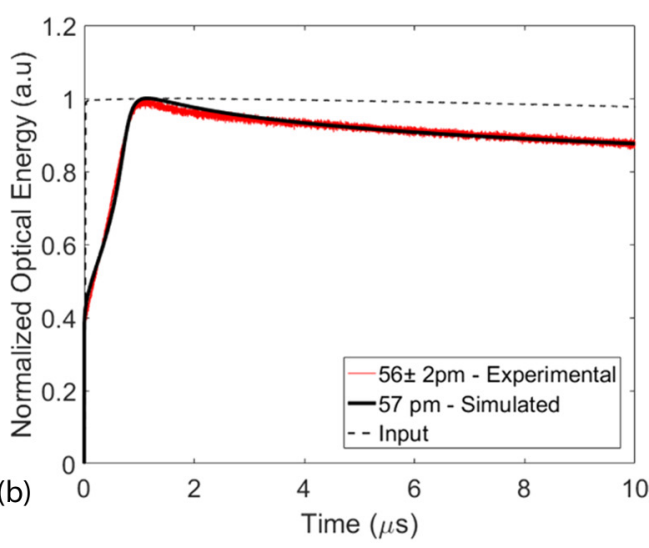

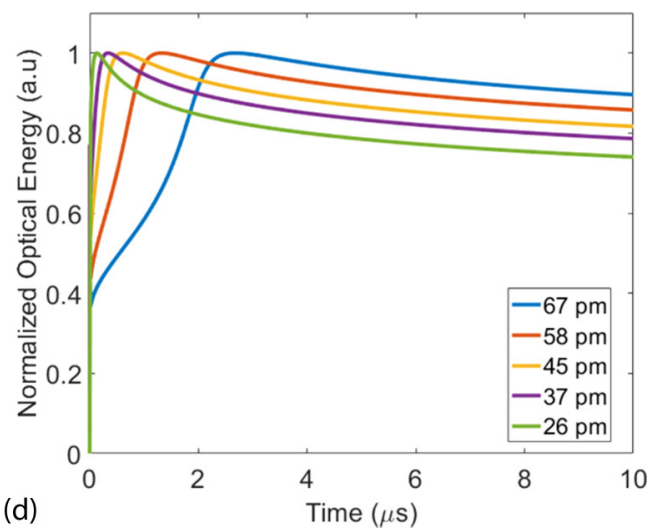

FIG. 6. (a) Optical mode confinement of a silicon $L 3 \mathrm{PhC}$ cavity calculated with finite difference time domain (FDTD) method. Red concentric ellipses represent a schematic of the spatial subdivisions used for the thermal calculation. (b) Plot of the calculated normalized optical energy in the $L 3$ cavity against time with $\lambda_{\text {Laser }}-\lambda_{0}=55 \mathrm{pm}$ (black curve) superimposed to the experimental normalized optical energy collected from the $L 3$ cavity against time with same the laser-cavity detuning (purple curve), the black dashed line represents the experimental input signal. (c) Experimental dynamic response of the $L 3$ cavity: the normalized optical energy collected from the DA cavity is plotted against time. The different colored curves represent the cavity response with different laser-cavity detuning $\lambda_{\text {Laser }}-\lambda_{0}$, the black dashed line represents the experimental input signal. (d) Dynamics of the DA cavity calculated with the model described in this work. The different laser-cavity detuning $\lambda_{\text {Laser }}-\lambda_{0}$ is matched to the experimental measurements for the calculations. Normalization has been carried out by dividing each raw curve by its maximum value, so that all the peaks were reaching unity.

the broader its temperature profile in space as the heat generated by the pump is being slowly dissipated. For the same reason, the increasingly more thermally insulated cavities are heating up faster, reaching higher temperatures and dissipat-

(a)

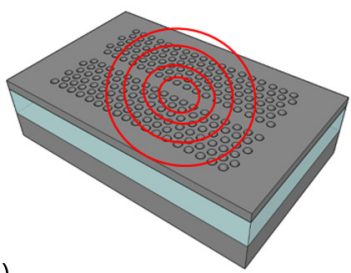

(b)

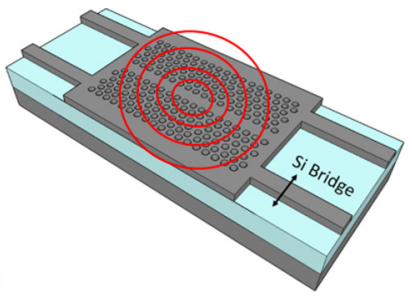

FIG. 7. (a) Schematics of a PhC cavity patterned on a silicon slab. (b) Schematics of the same PhC cavity patterned on silicon and connected to the rest of the silicon slab only through four small silicon bridges. Due to the very low thermal conductivity of air and the buried oxide, the $\mathrm{PhC}$ cavity in this configuration is transferring heat to the rest of the silicon slab less efficiently as the major heat transfer contribution is associated to the silicon bridges and their dimensions. The red concentric circumferences represent zones with equal temperatures. ing heat more slowly over time, as shown in Figs. 8(b) and 8(d).

The constantly expanding thermal volume over time shown in the plots confirms the need for the calculation of the system's space-time distribution of temperatures, as it has been provided with the model presented here, in order to accurately describe and design the long-time-scale thermodynamics of the pump-cavity problem.

\section{CONCLUSIONS}

We have presented a first principles model description for the physics of the thermo-optic nonlinear response of optically pumped silicon microcavities. The system temperature distribution is calculated both in space and time by using the Laplacian heat-transfer equation, which is then employed to model the thermo-optic response of the cavity on both fast $(\sim 100 \mathrm{~ns})$ and slow (steady state) time scales. By using a priori material parameters, this model has predictive power, even in the presence of time-varying input signals, allowing for the design of nonlinear optical behavior of optical microcavities, i.e., a powerful tool for novel, cavity based nonlinear optical devices. 

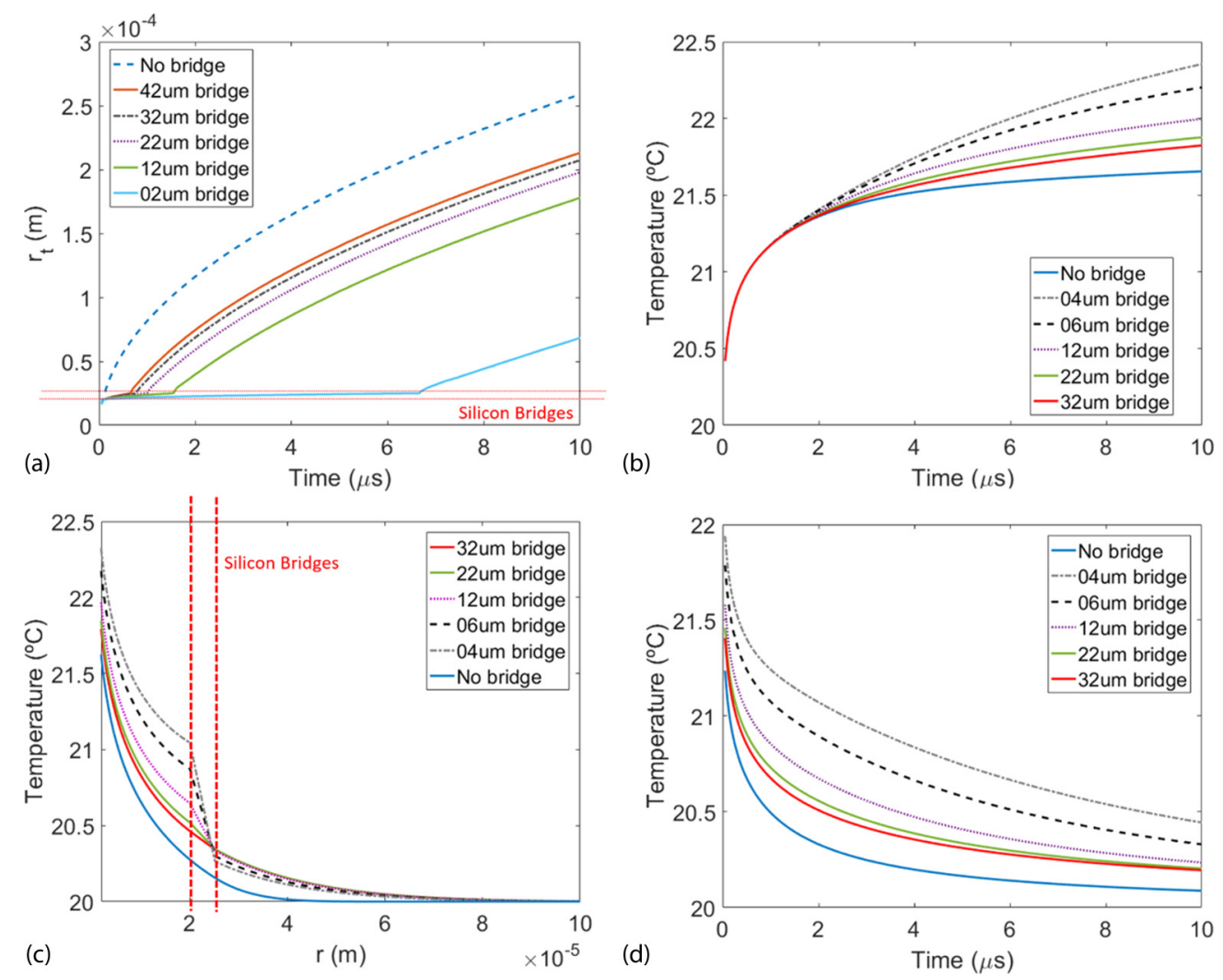

FIG. 8. (a) Calculated expanding heated volume of the DA cavity: the spatial coordinate at which the temperature of the material decays to $1 / e^{2}\left(r_{t}\right)$ is plotted against time. Different colors represent different cavity configurations: the blue curve is for the PhC patterned on the continuous slab of silicon while the other curves represent $\mathrm{PhC}$ cavities connected to the silicon slab by silicon bridges of decreasing width: $42,32,22,12$, and $2 \mu \mathrm{m}$ respectively, while the red dashed lines represent the location and delimitation in space of the silicon bridges in the different geometries. (b) Calculated temperature of the DA cavity with time for the different cavity configurations (continuous silicon slab—silicon bridges of decreasing widths: $32,22,12,6$, and $4 \mu \mathrm{m}$ respectively). (c) Temperature space profile $(r)$ of the silicon slab starting from the center of the DA cavity for the different $\mathrm{PhC}$ configurations, in which the silicon bridges locations are delimited with red dashed lines. (d) Temperature of the cavity with time when the laser is switched off at instant $t=0 \mathrm{~s}$ for the different PhC configurations.

\section{ACKNOWLEDGMENTS}

S.I., C.H., and L.O. acknowledge funding from the Science Foundation Ireland (17/QERA/3472, 12/RC/2276_P2) and in part by the European Research Council Starting Grant 337508 (DANCER) and under Grant 780240 (REDFINCH). M.C., D.G., and M.G. acknowledge the Horizon 2020 Framework Programme (H2020) through the QuantERA ERA-NET Cofund in Quantum Technologies, project CUSPIDOR, cofunded by Ministero dell'Istruzione, dell'Università e della Ricerca (MIUR), and MIUR through the "Dipartimenti di Eccellenza Program (2018-2022)", Department of Physics, University of Pavia.

\section{APPENDIX}

\section{Lorentzian derivation}

The optically pumped $\mathrm{PhC}$ cavity can be treated mathematically as a resonator with an input wave or waveguide using temporal coupled mode theory (CMT), e.g., following Ref. [46]. In this approach, the time-dependent cavity mode amplitude $a(t) \propto e^{j \omega_{0} t} e^{-t / \tau_{0}}$ with a resonance frequency $\omega_{0}$ has to be modified to take into account not only the total internal (i.e., absorption or scattering) losses, represented by the time-decay with constant $\tau_{0}$, but also the power coupled to the waveguide/external field and the possible excitation by an incident wave.

The rate of change of the mode amplitude, $d a(t) / d t$, considering coupling out of the cavity, characterized by a time constant $\tau_{\text {out }}$, and excitation by a traveling wave of amplitude $s_{+} \propto j \omega t$ incident on the cavity, becomes

$$
\frac{d a(t)}{d t}=j \omega_{0} a(t)-\left(\frac{1}{\tau_{0}}+\frac{1}{\tau_{\mathrm{out}}}\right) a(t)+\kappa s_{+} .
$$

Here $\kappa$ is the coupling coefficient between the cavity and the incident wave, and $s_{+}$is normalized on the incident power, i.e., $\left|s_{+}\right|^{2}=P_{0}$. The mode amplitude then becomes

$$
a=\frac{\kappa s_{+}}{j\left(\omega-\omega_{0}\right)+\left[\left(\frac{1}{\tau_{0}}\right)+\left(\frac{1}{\tau_{\text {out }}}\right)\right]} .
$$

To derive the expression for $\kappa$ time reversibility is applied, assuming the case of no internal losses $\left(1 / \tau_{0}=0\right)$, an initial starting amplitude $a$, and no source, i.e., $s_{+}=0$. The mode decays with the rate $1 / \tau_{\text {out }}$ with the power carried by the wave $s_{-}$, traveling away from the cavity. Due to energy 
conservation, and Eq. (A2), we find

$$
\frac{d|a|^{2}}{d t}=-\frac{2}{\tau_{\text {out }}}|a|^{2}=-\left|s_{-}\right|^{2} .
$$

When considering the time-reversed solution to Eq. (A3), the wave traveling away is converted into a wave traveling towards the cavity, i.e., $s_{+}$, and the energy builds up in the cavity with time dependence $\exp \left[\left(2 / \tau_{\text {out }}\right) t\right]$. Similar to Eqs. (A2) and (A3), and denoting the positive frequency amplitude of the time reversed solution with $\tilde{a}$, we have

$$
\frac{d|\tilde{a}|^{2}}{d t}=\frac{2}{\tau_{\text {out }}}|\tilde{a}|^{2} \text {. }
$$

The time reversed response is driven at a frequency that grows with the rate $1 / \tau_{\text {out }}$ from the driver wave frequency $\omega_{0}$, leading to $\omega=\omega_{0}+j / \tau_{\text {out }}$. Substituting this into Eq. (A2), we have

$$
|\tilde{a}|=\frac{\kappa \tilde{s_{+}}}{2 / \tau_{\text {out }}} .
$$

With $\left|\tilde{s_{+}}\right|^{2}=\left|s_{-}\right|^{2}$ and $|\tilde{a}(0)|^{2}=|a(0)|^{2}$, the coupling coefficient becomes

$$
\left|s_{+}\right|^{2}=\frac{2}{\tau_{\text {out }}}|a|^{2}=\frac{2}{\tau_{\text {out }}}|\tilde{a}|^{2}=\frac{2}{\tau_{\text {out }}}\left|\frac{\kappa \tilde{s_{+}}}{2 / \tau_{\text {out }}}\right|^{2} \rightarrow \kappa=\sqrt{\frac{2}{\tau_{\text {out }}}} .
$$

The rate of change of energy is therefore

$$
\begin{aligned}
\frac{d W}{d t} & =\frac{d a a^{*}}{d t}=\frac{a^{*} d a}{d t}+\frac{a d a^{*}}{d t} \\
& =-\left(\frac{2}{\tau_{\text {out }}}+\frac{2}{\tau_{0}}\right)|a|^{2}+\sqrt{\frac{2}{\tau_{\text {out }}}}\left(s_{+} a^{*}+s_{+}^{*} a\right),
\end{aligned}
$$

where $d W / d t$ is the rate of change of energy in the system. So, in the nonresonant condition, we have a mode amplitude $a_{\mathrm{NR}}(t)$ of the form

$$
a_{\mathrm{NR}}(t)=\frac{\sqrt{\frac{2}{\tau_{\mathrm{out}}}} e^{-j \omega_{0} t-\left[\left(\frac{1}{\tau_{\mathrm{out}}}\right)+\left(\frac{1}{\tau_{0}}\right)\right] t}}{-j\left(\omega-\omega_{0}\right)+\left[\left(\frac{1}{\tau_{\mathrm{out}}}\right)+\left(\frac{1}{\tau_{0}}\right)\right]}\left(s_{+}\right) .
$$

We define $\tau_{q}$ as the time constant associated to overall losses, extrapolated from the experimentally measured $Q$ factor, so that $\left(1 / \tau_{\text {out }}\right)+\left(1 / \tau_{0}\right)=1 / \tau_{q}$. Therefore Eq. (A8) can be written as

$$
a_{\mathrm{NR}}(t)=\frac{\sqrt{\frac{2}{\tau_{\mathrm{out}}}} e^{-j \omega_{0} t-\left[\left(\frac{1}{\tau_{q}}\right)\right] t}}{-j\left(\omega-\omega_{0}\right)+\left[\left(\frac{1}{\tau_{q}}\right)\right]}\left(s_{+}\right) .
$$

Replacing $a^{*}$ and $a$ into the coupling term in Eq. (A7) and applying properties of complex numbers we get

$$
\sqrt{\frac{2}{\tau_{\text {out }}}}\left(s_{+} a^{*}+s_{+}^{*} a\right)=2 \frac{\left(\frac{2}{\tau_{\text {out }}}\right)\left(\frac{1}{\tau_{q}}\right)}{\left(\omega-\omega_{0}\right)^{2}+\left(\frac{1}{\tau_{q}}\right)^{2}}\left|s_{+}\right|^{2},
$$

where, by definition, $\left|s_{+}\right|^{2}$ is the laser pump power incident on the cavity, $P_{0}$, with a driving frequency $\omega=\omega_{\text {laser }}$. Equation (A10) represents the case in which the frequency of the cavity mode $\omega_{0}$ is constant and the cavity is not affected by the pumping. In the case of a nonlinear cavity, however, the

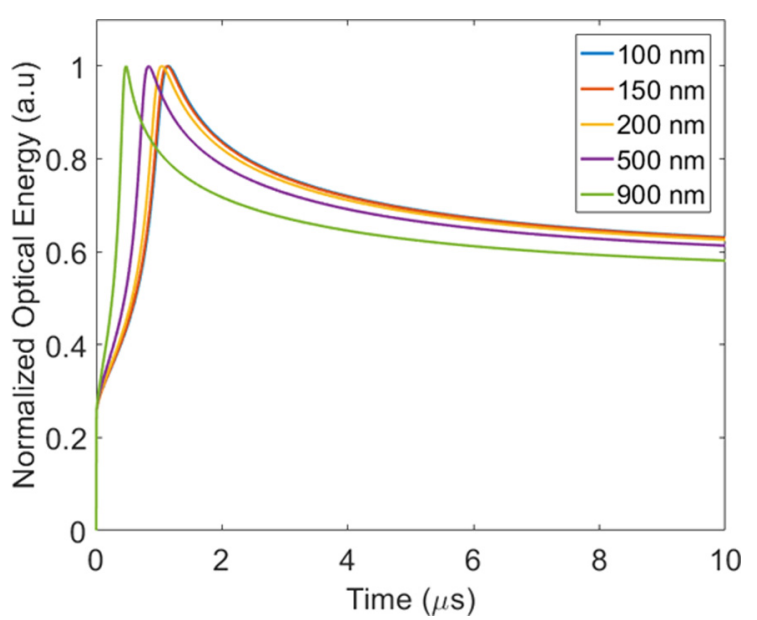

FIG. 9. Normalized optical energy in the DA cavity against time. The different colored curves represent calculations with exactly the same conditions but a different value of the increment $\Delta x$ into which the space of the simulation was divided.

pump power absorbed by the cavity through the linear and nonlinear absorption processes described earlier will shift the cavity resonance. Thus the Lorentzian term has to be modified with an effective cavity resonance frequency $\omega_{\text {eff }}$, in place of $\omega_{0}$, that takes into account the frequency shift due to the pump induced refractive index change. We obtain this term by converting Eq. (1) to the frequency

$$
\omega_{\text {eff }}=\omega_{0}\left[1-\frac{\Delta n_{\text {total }}}{n}\right] .
$$

The Lorentzian in Eq. (3) (coupling Lorentzian) then becomes

$$
L=2 * \frac{\left(\frac{2}{\tau_{\text {out }}}\right)\left(\frac{1}{\tau_{q}}\right)}{\left(\Delta \omega-\frac{\Delta n_{\text {total }}}{n} \omega_{0}\right)^{2}+\left(\frac{1}{\tau_{q}}\right)^{2}},
$$

with $\Delta \omega=\omega_{0}-\omega_{\text {laser }}$.

\section{Convergence tests}

We validated the resolution of the computations with various convergence tests, by scanning different values of the spatial and temporal increments to verify consistent results while optimizing the speed of the calculations. First, we performed a test on the concentric ellipses representing the enlarging thermal volume in time, by changing the value of the increment $\Delta x$ (see Fig. 9).

The calculations gave consistent results for $\Delta x=100 \mathrm{~nm}$ and $\Delta x=150 \mathrm{~nm}$ while bigger increments provide a too course spatial division of the system and temperature distribution, leading to the shifting curves shown in Fig. 9.

Then, we performed a convergence test to evaluate the temporal resolution needed for the calculations, by computing the dynamics of the optically pumped DA cavity with the exactly same conditions but changing the value of the time step used in the simulations. As Fig. 10(a) shows, a time-step range between 10 and 90 ps gives consistent results, highlighted 

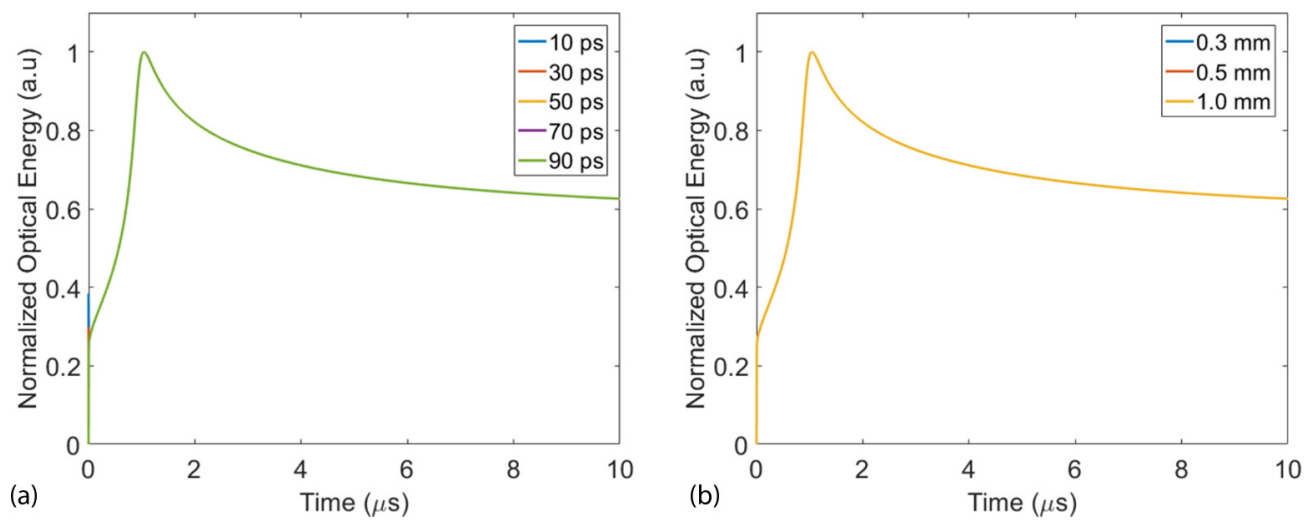

FIG. 10. (a) Normalized optical energy in the DA cavity against time: the different superimposed curves represent the calculations in the same conditions but different values of the time step into which the time of the simulation was divided. (b) Normalized optical energy in the DA cavity against time, the different superimposed curves represent the calculations in the same conditions but different total simulated space.

by the completely superimposed curves. The computations were also tested in order to verify that the total simulation area was large enough to ensure a realistic temperature decay from the cavity region to the room temperature bath (i.e., the rest of the sample). The perfectly overlapped curves in Fig. 10(b) demonstrate that setting the space of the simulation to $300 \mu \mathrm{m}$, as in the calculations showed in Figs. $4-6$, is enough to completely resolve the dynamics of the laser-cavity system while maintaining a higher simulation speed.

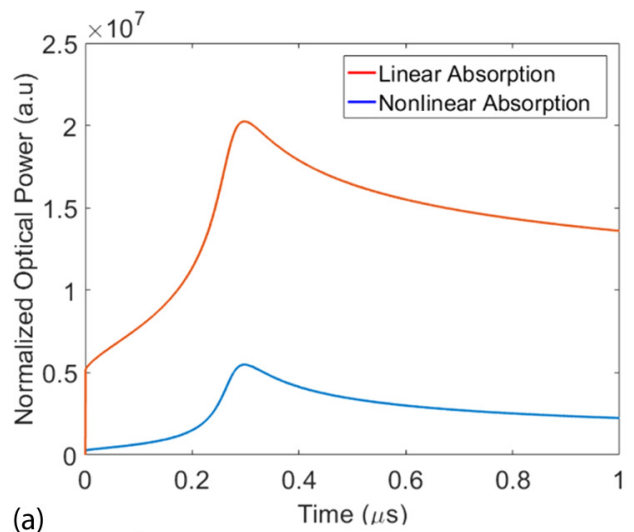

(a)

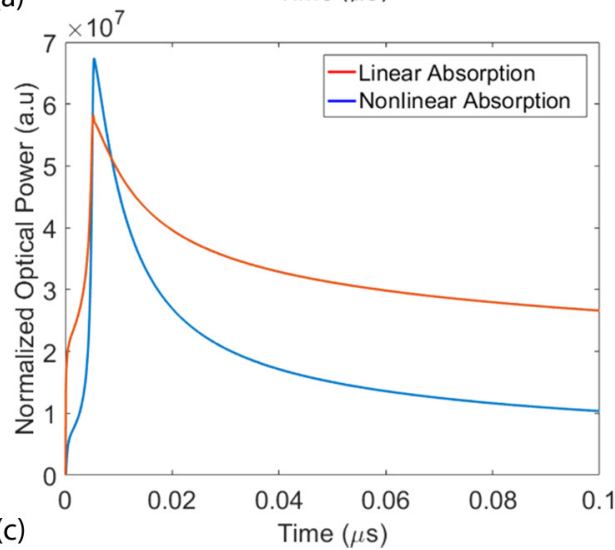

\section{Nonlinear dynamics}

Figure 4 shows experimental results and respective computations of the resonance wavelength shift against the power coupled in the DA cavity. The figure shows that in the coupled power range studied in the experiments the nonlinear absorption effects (TPA and FCA) are still very weak and the dominant mechanism in this regime is the linear absorption, most probably related to defects and surface states of the system. In this section, we show that the calculated nonlinearities

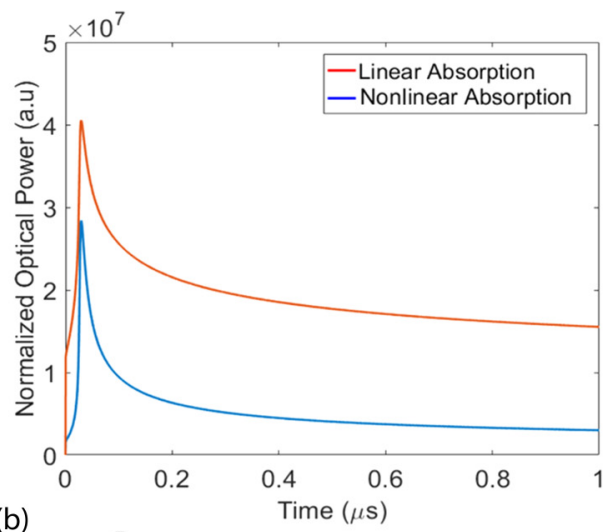

(b)

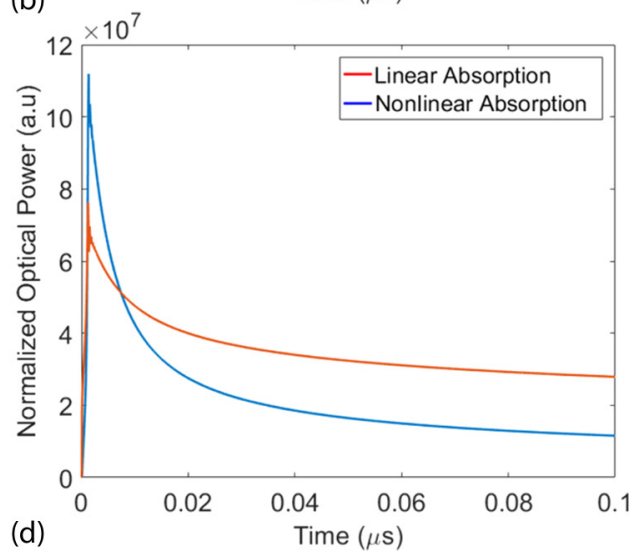

FIG. 11. Comparisons between linear absorption and nonlinear absorption related to TPA and FCA contributions to the dynamics of the optically pumped DA cavity. Each of the four plots represents the heat generated (in pJ) in the cavity by linear absorption (red) and nonlinear absorption (blue) against time with different coupled power: (a) $16 \mu \mathrm{W}$, (b) $25 \mu \mathrm{W}$, (c) $43 \mu \mathrm{W}$, (d) $70 \mu \mathrm{W}$. 

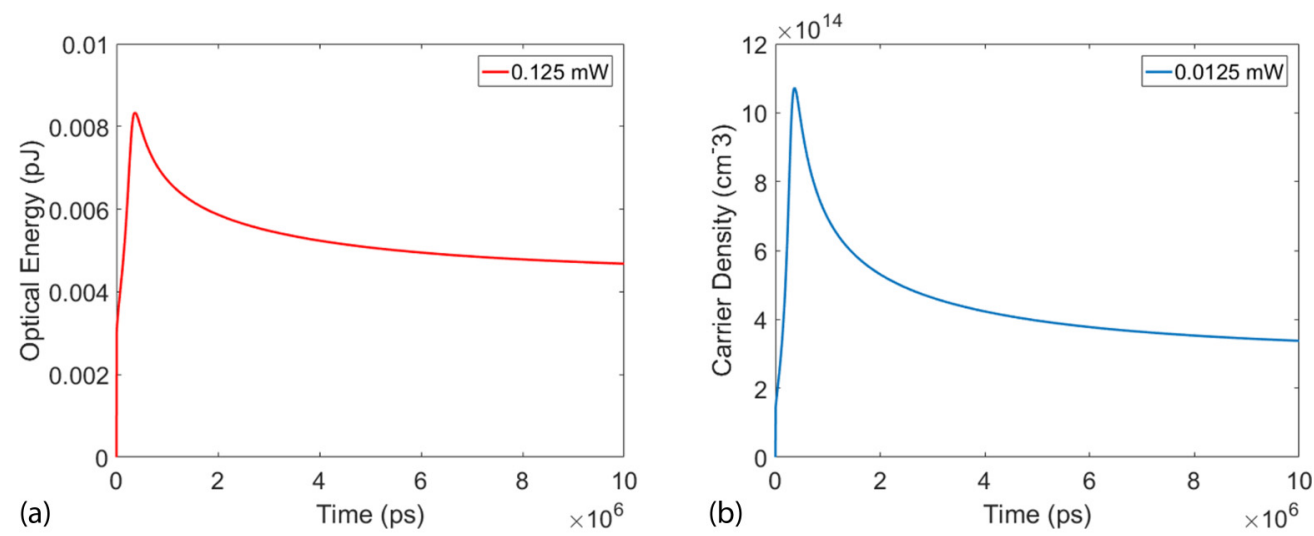

FIG. 12. (a) Optical energy in the DA cavity with time, with a peak value corresponding to $u_{0}=0.0085 \mathrm{pJ}$ and (b) free carrier density with time, with a peak value corresponding to $N_{0}=1.072 \times 10^{15} \mathrm{~cm}^{-3}$.

related to TPA and FCA become more and more dominant when the optical power coupled in the cavity increases beyond the range discussed earlier in this work.

Figure 11 shows the contributions to the heat generated in the cavity by linear and nonlinear absorption (TPA and FCA combined) in time, with increasing optical power coupled into the cavity: specifically $16,25,43$, and $70 \mu \mathrm{W}$. The plots clearly convey the fact that nonlinearities as the coupled power increases the nonlinear mechanism first become comparable to and then (for short time scales) dominate over the linear absorption process.

\section{Linear absorption, FCA, and TPA contributions to the cavity losses}

In this section we show calculations to quantify the contribution of free carrier absorption (FCA) to the total absorption in the experimental case considered, in order to validate the neglecting of the FCA influence on the cavity $Q$ factor in the model.

To determine the relative contributions, we look at the decay of optical energy in the cavity in different scenarios. The cavity is designed for strong outcoupling, which is by far the dominant effect, with linear absorption next. As shown below, FCA and TPA have very small effects on the $Q$ factor, and we feel justified in ignoring their effects, at least at the power levels considered here.

First, we used the model to get the DA cavity response dynamics for one of the detuning cases discussed in this work (31 pm), with an incident power of $0.125 \mathrm{~mW}$ (in the range of values used in this work). From this response we collected the peak optical energy in the cavity $\left[u\left(t_{\text {peak }}\right)=u_{0}\right]$ and the peak free carrier concentration values $\left(N_{0}\right)$ (Fig. 12).

These values are then used to simulate the optical energy decay in the cavity [i.e., $u(t=0)=u_{0}$ with no pumping], separating the contribution from linear absorption, FCA, and TPA (Fig. 3).

To get the optical energy decay curves in the cavity with and without nonlinear effects, we used the following equations (see Fig. 13):

$$
\begin{aligned}
\frac{d u(t)}{d t}= & -u(t)\left(\frac{1}{\tau_{0}}+\frac{1}{\tau_{\text {in }}}+\frac{1}{\tau_{\text {lin }}}\right)-\left(\frac{c}{n}\right) \sigma_{\mathrm{FCA}} u(t) N_{0} \\
& -\frac{\beta}{V_{\text {mode }}}\left(\frac{c}{n}\right)^{2} u^{2}(t),
\end{aligned}
$$
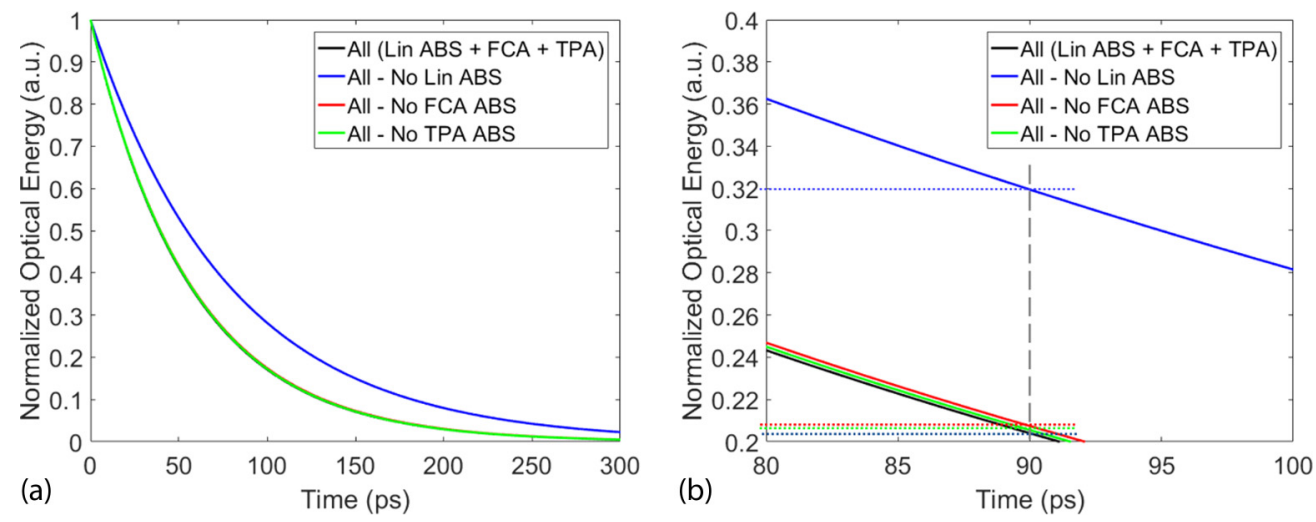

FIG. 13. (a) Optical energy decay in the DA cavity in different cases: linear and nonlinear losses (blue), no linear absorption (black), no free-carrier absorption (green), and no two-photon absorption (red). (b) Zoom of (a) to highlight the FCA and TPA contribution to the optical energy decay. The dashed lines show the values at which the different decays reach the 1/e value. Linear absorption contributes about ten times more to the losses compared to FCA and TPA in this power regime. 
where

$$
-u(t)\left(\frac{1}{\tau_{0}}+\frac{1}{\tau_{\text {in }}}+\frac{1}{\tau_{\text {lin }}}\right)=-\frac{u(t)}{\tau_{q}}
$$

with $\tau_{q}=57.742$ ps representing the characteristic time related to the total cavity losses, and corresponding to the measured $Q$ factor of $35000, \tau_{\text {lin }}=200$ ps representingw the linear absorption used in the calculation (estimated from Fig. 4 in the main text), $\tau_{i n} \sim 90$ ps represents the characteristic time related the coupling of energy into the cavity, derived from the experimentally estimated coupling efficiency. $\tau_{0} \sim 730 \mathrm{ps}$ is the characteristic time of the intrinsic cavity losses (with a $Q$ factor of $\sim 450000$ ).

The first term of Eq. (A14) represents the optical energy decay due to linear losses while the second and third terms express the decay due to FCA and TPA respectively. Figure 2 shows that in this power range, the dominant contribution to the cavity losses besides the loaded $Q$ factor is linear absorption, while free-carrier absorption plays a relatively weak role-FCA and TPA combined are about 1/10 of the linear absorption. Hence, the FCA influence on the cavity linewidth can be neglected without compromising quantitative capture of the cavity dynamics.
[1] R. J. Liu, L. Liao, D. S. Rubio, D. Rubin, O. Cohen, R. Nicolaescu, and M. Paniccia, A high-speed silicon optical modulator based on a metal-oxide-semiconductor capacitor, Nature (London) 427, 615 (2004).

[2] S. Rumley, D. Nikolova, R. Hendry, Q. Li, D. Calhoun, and K. Bergman, Silicon photonics for exascale systems, J. Lightwave Technol. 33, 547 (2015).

[3] A. H. Atabaki, S. Moazeni, F. Pavanello, H. Gevorgyan, J. Notaros, L. Alloatti, M. T. Wade, C. Sun, S. A. Kruger, H. Meng, K. Al Qubaisi, I. Wang, B. Zhang, A. Khilo, C. V. Baiocco, M. A. Popović, V. M. Stojanović, and R. J. Ram, Integrating photonics with silicon nanoelectronics for the next generation of systems on a chip, Nature (London) 556, 349 (2018).

[4] F. Dell'Olio and V. M. N. Passaro, Optical sensing by optimized silicon slot waveguides, Opt. Express 15, 4977 (2007).

[5] R. Janeiro, R. Flores, and J. Viegas, Silicon photonics waveguide array sensor for selective detection of VOCs at room temperature, Sci. Rep. 9, 17099 (2019).

[6] V. B. Braginsky, M. L. Gordetsky, and V. S. Ilchenko, Qualityfactor and nonlinear properties of optical whispering-gallery modes, Phys. Lett. A 137, 393 (1989).

[7] D. Armani, T. Kippenberg, S. Spillane, and K. J. Vahala, Ultrahigh- $Q$ toroid microcavity on a chip, Nature (London) 421, 925 (2003).

[8] V. S. Ilchenko, M. L. Gorodetsky, X. S. Yao, and L. Maleki, Microtorus: A high-finesse microcavity with whispering-gallery modes, Opt. Lett. 26, 256 (2001).

[9] A. Simbula, M. Schatzl, L. Zagaglia, F. Alpeggiani, L. C. Andreani, F. Schäffler, T. Fromherz, M. Galli, and D. Gerace, Realization of high-Q/V photonic crystal cavities defined by an effective aubry-andré-harper bichromatic potential, APL Photonics 2, 056102 (2017).

[10] Y. Akahane, T. Asano, B.-S. Song, and S. Noda, Fine-tuned high-Q photonic-crystal nanocavity, Opt. Express 13, 1202 (2005).

[11] Q. Quan and M. Loncar, Deterministic design of wavelength scale, ultra-high Q photonic crystal nanobeam cavities, Opt. Express 19, 18529 (2011).

[12] S. L. Portalupi, M. Galli, C. Reardon, T. F. Krauss, L. O'Faolain, L. C. Andreani, and D. Gerace, Planar photonic crystal cavities with far-field optimisation for high coupling efficiency and quality factor, Opt. Express 18, 16064 (2010).
[13] K. Welna, S. L. Portalupi, M. Galli, L. O'Faolain, and T. F. Krauss, Novel dispersion-adapted photonic crystal cavity with improved disorder stability, IEEE J. Quantum Electron. 48, 1177 (2012).

[14] Y. Tanaka, T. Asano, and S. Noda, Design of photonic crystal nanocavity with Q-factor of $\sim 10^{9}$, J. Lightwave Technol. 26, 1532 (2008).

[15] M. Notomi, A. Shinya, S. Mitsugi, E. Kuramochi, and H-Y. Ryu, Waveguides, resonators and their coupled elements in photonic crystal slabs, Opt. Express 12, 1551 (2004).

[16] K. Debnath, K. Welna, M. Ferrera, K. Deasy, D. G. Lidzey, and L. O'Faolain, Highly efficient optical filter based on vertically coupled photonic crystal cavity and bus waveguide, Opt. Lett. 38, 154 (2013).

[17] K. Hennessy, A. Badolato, M. Winger, D. Gerace, M. Atatüre, S. Gulde, S. Fält, E. L. Hu, and A. Imamoğlu, Quantum nature of a strongly coupled single quantum dot-cavity system, Nature (London) 445, 896 (2007).

[18] S. F. Preble, V. R. Almeida, and M. Lipson, Optically controlled photonic crystal nanocavity in silicon, Proc. SPIE 5511, 10 (2004).

[19] S. Iadanza, C. G. R. Devarapu, A. A. Liles, R. Sheehan, and L. O'Faolain, Hybrid external cavity laser with an amorphous silicon-based photonic crystal cavity mirror, Appl. Sci. 10, 240 (2020).

[20] A. P. Bakoz, A. A. Liles, A. A. Gonzalez-Fernandez, T. Habruseva, C. Hu, E. A. Viktorov, S. P. Hegarty, and L. O'Faolain, Wavelength stability in a hybrid photonic crystal laser through controlled nonlinear absorptive heating in the reflector, Light Sci. Appl. 7, 39 (2018).

[21] T. J. Kippenberg, S. M. Spillane, and K. J. Vahala, KerrNonlinearity Induced Optical Parametric Oscillation in a Ultra-High-Q Toroid Microcavity, Phys. Rev. Lett. 93, 083904 (2004).

[22] M. Soljacic and J. Joannopoulos, Enhancement of nonlinear effects using photonic crystals, Nat. Mater. 3, 211 (2004).

[23] R. K. Chang and A. J. Campillo, Optical Processes in Microcavities (World Scientific, Singapore, 1996).

[24] M. Galli, D. Gerace, K. Welna, T. F. Krauss, L. O'Faolain, G. Guizzetti, and L. C. Andreani, Low-power continuous-wave generation of visible harmonics in silicon photonic crystal nanocavities, Opt. Express 18, 26613 (2010). 
[25] V. R. Almeida and M. Lipson, Optical bistability on a silicon chip, Opt. Lett. 29, 2387 (2004).

[26] T. Tanabe, M. Notomi, S. Mitsugi, A. Shinya, and E. Kuramochi, Fast bistable all-optical switch and memory on a silicon photonic crystal on-chip, Opt. Lett. 30, 2575 (2005).

[27] T. Carmon, L. Yang, and K. J. Vahala, Dynamical thermal behavior and thermal self-stability of microcavities, Opt. Express 12, 4742 (2004).

[28] V. S. Ilchenko and M. L. Gorodetskii, Thermal nonlinear effects in optical whispering gallery microresonators, Laser Phys. 2, 1004 (1992).

[29] M. Galli, S. L. Portalupi, M. Belotti, L. C. Andreani, L. O'Faolain, and T. F. Krauss, Light scattering and Fano resonances in high-photonic crystal nanocavities, Appl. Phys. Lett. 94, 071101 (2009).

[30] N-Vi-Q. Tran, S. Combrié, and A. De Rossi, Directive emission from high-Q photonic crystal cavities through band folding, Phys. Rev. B 79, 041101(R) (2009).

[31] P. Becker, P. Scyfried, and H. Siegert, The lattice parameter of highly pure silicon single crystals, Z. Phys. B 48, 17 (1982).

[32] J. Komma, C. Schwarz, G. Hofmann, D. Heinert, and R. Nawrodt, Thermo-optic coefficient of silicon at $1550 \mathrm{~nm}$ and cryogenic temperatures, Appl. Phys. Lett. 101, 041905 (2012).

[33] R. Soref and B. Bennett, Electrooptical effects in silicon, IEEE J. Quantum Electron. 23, 123 (1987).

[34] A. D. Bristow, N. Rotenberg, and H. M. van Driel, Two-photon absorption and Kerr coefficients of silicon for 850-2200 nm, Appl. Phys. Lett. 90, 191104 (2007).

[35] S. Ramanujan and G. H. Hardy, Ramanujan's Collected Works (Chelsea, New York, 1962).
[36] C. J. Glassbrenner and G. A. Slack, Thermal Conductivity of Silicon and Germanium from $3^{\circ} \mathrm{K}$ to the Melting Point, Phys. Rev. 134, A1058 (1964) .

[37] J. C. Thompson and B. A. Younglove, Thermal conductivity of silicon at low temperatures, J. Phys. Chem. Solids 20, 146 (1961).

[38] A. S. Okhotin, A. S. Pushkarskii, and V. V. Gorbachev, Thermophysical Properties of Semiconductors (Atom Publ. House, Moscow, 1972).

[39] J. Yu, S. Mitrovic, D. Tham, J. Varghese, and J. Heath, Reduction of thermal conductivity in phononic nanomesh structures, Nat. Nanotechnol. 5, 718 (2010).

[40] A. Jain, Y. Yu, and A. McGaughey, Phonon transport in periodic silicon nanoporous films with feature sizes greater than $100 \mathrm{~nm}$, Phys. Rev. B 87, 195301 (2013).

[41] N. Zen, T. Puurtinen, T. Isotalo, S. Chaudhuri, and I. Maasilta, Engineering thermal conductance using a two-dimensional phononic crystal, Nat. Commun. 5, 3435 (2014).

[42] A. S. Grove, Physics and Technology of Semiconductor Devices (Wiley, New York, 1967).

[43] W. H. Haynes, D. R. Lide, and T. J. Bruno, CRC Handbook of Chemistry and Physics, A Ready-reference Book of Chemical and Physical Data, Ninety-seven, ed. (CRC Press, Boca Raton, London, 2017).

[44] L. O'Faolain, S. A. Schulz, D. M. Beggs, T. P. White, M. Spasenović, L. Kuipers, F. Morichetti, A. Melloni, S. Mazoyer, J. P. Hugonin, P. Lalanne, and T. F. Krauss, Loss engineered slow light waveguides, Opt. Express 18, 27627 (2010).

[45] A. Di Falco, L. O'Faolain, M. Lapine, P. Tassin, and M. Vanwolleghem, Photonics Nanostruct: Fund. Appl. 10, 589 (2012).

[46] H. A. Haus, Waves and Fields in Optoelectronics (Prentice-Hall, Englewood Cliffs, NJ, 1984). 